Pediat. Res. 2: 43-63 (1968)

Dwarfism, constitutional hypoglycemia dwarfism, psychosocial insulin gonadal dysgenesis growth hormone growth retardation maternal depriva-

tion syndrome

panhypopituitarism

\title{
Growth and Growth Hormone
}

\author{
I. Changes in Serum Level of Growth Hormone Following Hypoglycemia in 134 Children
} with Growth Retardation

\author{
S.L.Kaplan, C.A.L.Abrams, J.J.Bell, F.A. Conte and M.M.Grumbach ${ }^{[41]}$ \\ Department of Pediatrics, University of California, San Francisco Medical Center, San Francisco, California, \\ and College of Physicians and Surgeons, Columbia University, New York, New York, USA
}

Extract

The change in levels of growth hormone in serum (SGH) following insulin-induced hypoglycemia was evaluated in 134 prepubertal children with growth retardation and 10 control subjects with normal growth patterns by radioimmunoassay, utilizing ${ }^{131} \mathrm{I}-\mathrm{HGH}$ and rabbit antiserum to human growth hormone. Mean maximum growth hormone concentration $(\mathrm{m} \mu \mathrm{g} / \mathrm{ml})$ at any time during the test was:

$\begin{array}{lc}\text { 10 Control subjects } & 12.4 \\ \text { 53 Hypopituitarism } & 2.5 \mathrm{~m} \mu \mathrm{g} \text { or less in } 52 / 53 \\ \text { 20 Constitutional shortness of stature } & 12.5 \\ \text { 22 Primordial dwarfism } & 12.1 \\ 9 \text { XO gonadal dysgenesis } & 13.4 \\ \text { 5 Delayed adolescence } & 11.8 \\ 5 \text { Maternal deprivation } & 16.7 \\ 9 \text { Psychosocial dwarfism } & 10.9 \\ 11 \text { Miscellaneous disorders } & 7.0\end{array}$

Among factors found to affect the SGH response to insulin-induced hypoglycemia were: a) elevated fasting concentration of SGH which appeared to alter the responsiveness to stimulation; and b) age.

The mean maximum SGH concentration of the control subjects following insulin-induced hypoglycemia was $12.4 \mathrm{~m} \mu \mathrm{g} / \mathrm{ml}$.

In 52/53 patients with hypopituitarism, the SGH concentration was $1 \mathrm{~m} \mu \mathrm{g}$ or less at rest, with no increase or an increase to a maximum of $2.5 \mathrm{~m} \mu \mathrm{g} / \mathrm{ml}$ following hypoglycemia. One patient (CL) had a fasting serum growth hormone (FSGH) concentration of $3.4 \mathrm{~m} \mu \mathrm{g} / \mathrm{ml}$ with a rise to $5 \mathrm{~m} \mu \mathrm{g} / \mathrm{ml}$ at 60 minutes, as indicated in tables II and II a.

Clinical data on 20 patients with constitutional shortness of stature are presented in table III. The mean maximum $\mathrm{GH}$ concentration following insulin-induced hypoglycemia of this group was $12.5 \mathrm{~m} \mu \mathrm{g} / \mathrm{ml}$, a value comparable to that obtained in the control subjects (table III a).

Clinical data, including growth rate, of patients with primordial dwarfism are presented in table IV. The mean maximum SGH concentration following hypoglycemia of the 22 patients in this group was $12.1 \mathrm{~m} \mu \mathrm{g} / \mathrm{ml}$ (table IV a). This response was not significantly different from that observed in the control group. 
Nine patients with $\mathrm{XO}$ gonadal dysgenesis had a mean maximum SGH response to hypoglycemia of $13.4 \mathrm{~m} \mu \mathrm{g} / \mathrm{ml}$, as indicated in tables VI and VIa.

Five children with delayed adolescence had a mean maximum SGH response of $11.8 \mathrm{~m} \mu \mathrm{g} / \mathrm{ml}$, as shown in tables VI and VIa.

Four of five infants with maternal deprivation included in this study showed evidence of increased insulin sensitivity, but the mean maximum SGH response was not significantly different from that of the control group. Clinical data on nine patients with psychosocial dwarfism are presented in table VI. The nine children in this group had a mean maximal rise of SGH concentration to $10.9 \mathrm{~m} \mu \mathrm{g} / \mathrm{ml}$. Two of the children had an abnormal SGH response with concentrations of $1 \mathrm{~m} \mu \mathrm{g} / \mathrm{ml}$ in the fasting specimen and no rise following hypoglycemia. RS, who was retested after a hospitalization period of two months, had a maximum SGH rise to $7.4 \mathrm{~m} \mu \mathrm{g} / \mathrm{ml}$ (table VIa).

Clinical data on 11 patients with a variety of diseases associated with their growth retardation are presented in table VII. Included in this group are two males, NF and MB, with blunted responses to insulin-induced hypoglycemia and in whom the diagnosis of partial GH deficiency cannot be excluded.

As indicated on figures $\mathrm{I} a$ and $\mathrm{lb}$, the mean maximum SGH response to insulin-induced hypoglycemia in children with growth retardation was not significantly different from the response observed in the control group. There was, however, a highly significant difference in the response of the control group when compared to the group of children with hypopituitarism.

In the presence of an elevated SGH concentration, eight of the eighteen subjects showed a decrease in FGH concentration or no response following insulin-induced hypoglycemia. This was observed in four control subjects and four children with growth retardation. The level of FSGH was significantly higher in children less than four years of age, but there was no significant correlation of the level of FSGH with age in children over four years, or according to sex. In this study, there was no correlation demonstrated between a decrease of $40 \%$ or greater in blood glucose and the maximum GH response achieved.

The response to therapy with human pituitary growth hormone (HGH) was evaluated in 8 children with growth retardation who had a mean maximum SGH concentration of $12.0 \mathrm{~m} \mu \mathrm{g} / \mathrm{ml}$. In six of the patients, none of whom had the clinical features of pituitary dwarfism, there was no significant change in the rate of growth during the period of administration of $\mathrm{HGH}$. Three of the six patients subsequently had low levels of antibodies to HGH and three had no detectable antibodies. ER, a primordial dwarf, had an acceleration in his growth rate during the first period of treatment with HGH which was not sustained. An increase in growth rate to $6.9 \mathrm{~cm} / \mathrm{yr}$ was also observed in the second patient (NF) suspected of having mild or partial GH deficiency. In contrast, 20 patients with SGH levels of less than $2 \mathrm{~m} \mu \mathrm{g} / \mathrm{ml}$ had a growth rate of 9 to $15 \mathrm{~cm} / \mathrm{yr}$ on treatment with $\mathrm{HGH}$.

No evidence of a GH deficiency or a defect in the responsiveness of the hypothalamic GH-releasing mechanism was demonstrated in children with growth disorders, including constitutional shortness of stature, primordial dwarfism and gonadal dysgenesis. On the basis of our data, a FSGH of $7 \mathrm{~m} \mu \mathrm{g} / \mathrm{ml}$ or an increase to $7 \mathrm{~m} \mu \mathrm{g} / \mathrm{ml}$ or more following insulin-induced hypoglycemia has a high probability of association with normal pituitary acidophile function. A rigid definition of the normal range of SGH response to hypoglycemia cannot be established because, in a small proportion of instances, there is overlap between the normal subject and some GH-deficient patients.

Problems in the interpretation of the response to insulin-induced hypoglycemia have been observed. In children who have a borderline rise of SGH of 3 to $5 \mathrm{~m} \mu \mathrm{g} / \mathrm{ml}$, the response may be indicative of GH deficiency. In a few children, however, individual differences in responsiveness to various stimuli have been observed. It is suggested that GH stimulation tests with arginine infusion or vasopressin administration be used in a child who exhibits a blunted $\mathrm{GH}$ response to hypoglycemia. In some children who have an apparently normal serum 'immunoreactive' GH response to a provocative test despite physical stigmata of hypopituitarism, short-term therapy with HGH may be necessary to establish the diagnosis of GH insufficiency. 


\section{Speculation}

It has been observed that the changes in SGH induced by hypoglycemia are less in children than in adults. This difference between children and adults may be related to the concentration in serum of testosterone or estrogen, an altered sensitivity of the hypothalamus to stimuli in children, or to agerelated variations in the secretory rate of the pituitary. SGH response to insulin-induced hypoglycemia has been valuable in distinguishing children with GH deficiency, especially when this occurs as an isolated defect, from children with other forms of growth retardation. It has not been useful in identifying the etiology of growth retardation from other causes.

\section{Introduction}

Considerable speculation has been focused in the past on the role of growth hormone (GH) in growth disorders of children and the possibility that impairment or acceleration of growth in a variety of pathologic conditions may be mediated, at least in part, by affecting the secretion of pituitary GH. The development of sensitive, precise methods for the determination of immunoreactive GH in serum $[7,29,30]$ and of techniques to appraise the capacity of the anterior pituitary gland to secrete $\mathrm{GH}$ in response to certain stimuli (hypoglycemia, specific amino acids, exercise and fever) has made it possible to investigate the function of pituitary acidophils in disturbances of growth.

It has been established recently, largely through data obtained by radioimmunoassay, that secretion of human growth hormone ( $\mathrm{HGH})$ is provoked by a wide variety of stimuli $[4,5,7,13,14,17,22,29,30]$ and that it exhibits wide fluctuation in plasma concentration. These and other factors, especially the inadequate correlation of urinary excretion with plasma concentration, have posed major obstacles to the measurement of the rate of secretion of GH. As a consequence, attention has been focused on the utilization of provocative tests to assess the capacity of the pituitary to secrete GH. The relation between the 'homeostatic' actions of $\mathrm{GH}$ and its growth-promoting properties has not yet been clarified. The present report describes the results of studies of $\mathrm{GH}$ secretion in 134 children with short stature and the value and limitations of the insulin tolerance test in detection of impaired growth attributable to GH deficiency.

\section{Materials and Methods}

Patients. The changes in levels of growth hormone in serum (serum growth hormone [SGH]) in response to insulin-induced hypoglycemia was assessed in 134 prepubertal children whose growth retardation was 2 standard deviations (SD) or more below the mean for age. Ten prepubertal children with normal growth patterns (siblings of patients with short stature or subjects admitted for correction of minor orthopedic or genitourinary defects) were used as controls (table I).

The 53 patients with hypopituitarism included: 16 with isolated growth hormone deficiency; 19 with idiopathic hypopituitarism with multiple tropic hormone deficiencies; 15 with hypopituitarism secondary to an organic lesion of the pituitary or hypothalamus; and 3 with clinical evidence of hypopituitarism and diabetes insipidus, but no demonstrable tumor by roentgenograms of the skull and/or pneumoencephalogram (table II).

Patients with growth retardation were classified on the basis of established criteria [35], although it was recognized that each group might represent a heterogeneous mixture with respect to the etiology of the shortness of stature. The 20 children in the category of constitutional shortness of stature ranged from -2 to $-4 \mathrm{SD}$ below the mean of height for age; bone age was usually equivalent to height age and there was a positive family history of shortness of stature (table III).

The group of 22 with primordial dwarfism included a high proportion of children with intrauterine growth retardation. When studied, most were growing at a normal or near-normal rate for age, were markedly retarded in growth and had bone ages which were normal or retarded in varying degrees. The RussellSilver type and bird-headed dwarfs were also included in this group (table IV).

Nine patients had clinical stigmata of gonadal dysgenesis, a negative sex chromatin pattern demonstrated by buccal smear, and an XO chromosomal constitution. None had received any estrogen therapy (table V).

Five children with delayed adolescence were 13 years or older and had not shown signs of sexual development. They had retarded bone ages and a slow rate of growth during the previous year or two before evaluation (table V).

Five infants in the maternal deprivation group had a history of maternal neglect, poor feeding habits and malnutrition. They were cachectic, withdrawn, had evidence of psychomotor retardation and exhibited a rapid gain in weight during hospitalization (table VI). 
A group of 9 children with psychosocial dwarfism included those with bizarre eating and drinking habits, as well as other evidence of behavioral disorder and a disrupted family background [24]. The severity of the growth disorder varied, with a range in height of -4 to $-6.5 \mathrm{SD}$ below the mean for age. The bone age was usually less severely retarded. Characteristically, all showed a change in personality as well as an increase in growth rate during a period of separation from the home environment (table VI).

Eleven children with miscellaneous disorders associated with growth retardation did not fit the criteria used for the other categories (table VII).

Procedure. All patients were fasted overnight and kept at bed rest for 30 minutes before and during the period of collection of samples for the insulin tolerance test. In the group with psychosocial dwarfism, the insulin tolerance test was performed within 4 days after admission to the hospital.

Samples of blood were collected during the fasting state, at 15, 30, 45, 60, 90 and 120 minutes following intravenous administration of $0.1 \mathrm{U} / \mathrm{kg}$ of regular insulin. Venous blood samples were drawn through an indwelling needle attached to a 3-way stopcock with a slow infusion of $0.9 \%$ saline. In 18 patients, the sampling period was limited to 60 minutes; in 7 patients, the test was terminated because of symptoms of hypoglycemia.

Apart from those patients with gonadal dysgenesis, pituitary function was evaluated in 119 subjects with growth retardation. Thyroid stimulating hormone (TSH) function was assessed indirectly by measurement of serum protein-bound iodine (PBI). In those in whom the concentration of $\mathrm{PBI}$ was $<4 \mu \mathrm{g} / 100 \mathrm{ml}$, the TSH stimulation test was used.

ACTH function was evaluated indirectly in 50 patients by measurement of output of 17-hydroxycorticosteroid in urine in response to the oral administration of metyrapone [4, 27], $100 \mathrm{mg} / \mathrm{kg}$ daily, not exceeding $3 \mathrm{~g} /$ day, given at 4-hour intervals for 2 days [8]. In the remaining patients, only a single 24-hour excretion of urinary 17-hydroxycorticoids was determined.

The bone age was estimated from roentgenograms of the wrist and hand using the standards of GREULICH and Pyle [11]. The presence of an expanding lesion in the pituitary fossa or hypothalamus was evaluated by roentgenograms of the skull, delineation of visual fields and examination of the fundi. Height was expressed in $\mathrm{cm}$ as the average of 3 measurements. SD from mean value for age was used as the parameter for assessment and comparison of height [26].

Serum immunoreactive human growth hormone (HGH) was determined by the radioimmunoassay method of GLICK et al. [6] utilizing ${ }^{131}$ I-HGH and rabbit antiserum to HGH. Iodination was performed by the method of GreEnwood et al. [10], as modified by KAPLAN and GRUMBACH [16]. The ${ }^{131}$ I-HGH was purified by vertical starch gel electrophoresis using the discontinuous buffer system of FERGUSON and WALlace [1a], the specific activity of which varied between 280 and $340 \mu \mathrm{c} / \mu \mathrm{g}$. The 'damaged' iodination fraction represented $6-8 \%$ of the labeled product, as determined by the method of BERson et al. [1]. The sensitivity of the method permitted measurement of $0.05 \mathrm{~m} \mu \mathrm{g} / \mathrm{ml}$ of $\mathrm{HGH}$. Wilhelmi HGH (HS-612A, HS-705) was utilized as a reference standard. Blood glucose was determined by the glucose oxidase method [31].

\section{Results}

The maximum GH concentration ( $\mathrm{m} \mu \mathrm{g} / \mathrm{ml}$ ) for the 10 normal subjects is shown in figure $1 \mathrm{a}$, tables I and Ia.

The clinical data on 16 children with isolated growth hormone deficiency ${ }^{1}$ and 19 with idiopathic hypopituitarism with multiple tropic hormone deficiencies have been reported separately [8]; the data on the 18 patients with lesions of the hypothalamicpituitary region are presented in table II. Of the 15 with organic lesions, 2 had measurements of GH carried out both pre- and postoperatively; 5 had determinations only during the preoperative period and 8 only during the postoperative period.

In 52 of the 53 patients with hypopituitarism, the $\mathrm{SGH}$ concentration was $1 \mathrm{~m} \mu \mathrm{g}$ or less at rest, with no increase or an increase to a maximum of $2.5 \mathrm{~m} \mu \mathrm{g} / \mathrm{ml}$ following insulin-induced hypoglycemia. One (CL) had a fasting SGH concentration of $3.4 \mathrm{~m} \mu \mathrm{g} / \mathrm{ml}$, with a rise to 5 $\mathrm{m} \mu \mathrm{g} / \mathrm{ml}$ at 60 minutes - a distinctly blunted response. Three of the 12 patients with an organic lesion of the pituitary and evidence of normal TSH function grew at a rate of 4.1 to $7.2 \mathrm{~cm} /$ year, despite the low levels of serum immunoreactive growth hormone, which suggests that these patients secreted small amounts of $\mathrm{GH}$ even though they showed an abnormal response to the provocative tests (fig. 1 a, table II a).

Insulin sensitivity was demonstrable in 17 of the 21 patients with combined ACTH and HGH deficiency, but in only 8 of the 15 patients with isolated GH deficiency [8].

In the 20 patients with constitutional shortness of stature, the mean concentration of $\mathrm{GH}$ was $12.5 \mathrm{~m} \mu \mathrm{g} / \mathrm{ml}$ (fig. $1 \mathrm{a}$; tables III and IIIa), a value not significantly different from that of the control group, using the $t$ test applied to the means after logarithmic transformation of the data $(p>0.4)$. Despite a blunted response $(3.2$

${ }^{1}$ Limitations of this diagnosis in the prepubertal child are discussed in reference 8. 
$\mathrm{m} \mu \mathrm{g} / \mathrm{ml}$ ) to hypoglycemia, one patient (RR) showed a brisk rise $(37.5 \mathrm{~m} \mu \mathrm{g} / \mathrm{ml})$ following intravenous administration of arginine.

The mean concentration of SGH in the 22 patients in the group with primordial dwarfism was $12.1 \mathrm{~m} \mu \mathrm{g} / \mathrm{ml}$, a response not significantly different from that of the control group ( $p>0.5$ ) (fig. $1 \mathrm{a}$; tables IV and IVa).

The response $(13.4 \mathrm{~m} \mu \mathrm{g} / \mathrm{ml})$ to insulin-induced hypoglycemia in the group with gonadal dysgenesis did

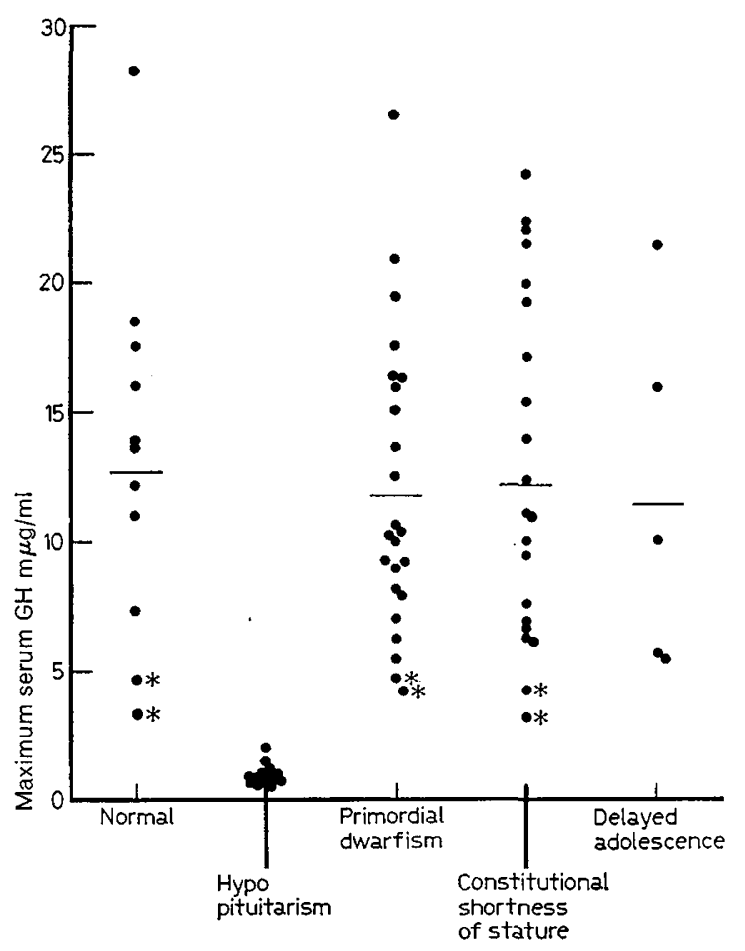

Fig. 1 1 . The maximum SGH concentration achieved following insulin-induced hypoglycemia is indicated as $\mathrm{m} \mu \mathrm{g} / \mathrm{ml}$. The horizontal line designates the mean level for each group. No significant difference is observed in the mean concentration of children with primordial dwarfism, constitutional shortness of stature, or delayed adolescence when compared with response of children of normal height. The SGH response of patients with hypopituitarism was significantly different from that of the control group. Maximum SGH concentrations in the borderline range are indicated by an asterisk (*). SC and $\mathrm{RD}$, in the control group, had elevated fasting levels; in primordial dwarfism group, $\mathrm{JM}$ had a SGH level of $22.3 \mathrm{~m} \mu \mathrm{g} / \mathrm{ml}$ following arginine infusion; DH will have arginine infusion in the future; in the group with constitutional shortness of stature, $\mathrm{RR}$ had a SGH concentration of $37.5 \mathrm{~m} \mu \mathrm{g} / \mathrm{ml}$ following arginine infusion; and RT has not been retested as yet.
Table 1. Clinical data of children with normal stature

\begin{tabular}{|c|c|c|c|c|c|}
\hline \multirow[t]{2}{*}{ Patient } & \multirow{2}{*}{$\begin{array}{l}\text { Age } \\
\text { years }\end{array}$} & \multicolumn{2}{|c|}{ Sex Height } & \multicolumn{2}{|c|}{ Level of SGH } \\
\hline & & & $\mathrm{cm}$ & $\begin{array}{l}\text { fasting } \\
\mathrm{m} \mu \mathrm{g} / \mathrm{ml}\end{array}$ & $\begin{array}{l}\operatorname{maximum} \\
\mathrm{m} \mu \mathrm{g} / \mathrm{ml}\end{array}$ \\
\hline BJ & $3-7 / 12$ & $\mathrm{~m}$ & 102.0 & 11.3 & 28.4 \\
\hline $\mathrm{SC}$ & $4-8 / 12$ & $\mathrm{~m}$ & 103.2 & 14.2 & 3.4 \\
\hline DB & $4-9 / 12$ & $f$ & 105.0 & 9.9 & 11.0 \\
\hline GG & $5-0 / 12$ & $f$ & 110.0 & 5.4 & 14.0 \\
\hline RD & $5-6 / 12$ & $f$ & 108.1 & 9.0 & 4.7 \\
\hline DK & $5-8 / 12$ & $f$ & 114.0 & 10.7 & 7.2 \\
\hline WJ & $6-9 / 12$ & $\mathrm{~m}$ & 122.5 & 2.6 & 11.0 \\
\hline $\mathrm{JG}$ & $8-0 / 12$ & $\mathrm{~m}$ & 131.2 & 2.0 & 13.6 \\
\hline IG & $10-6 / 12$ & $\mathrm{~m}$ & 138.0 & 2.9 & 12.2 \\
\hline NA & $13-4 / 12$ & $f$ & 156.0 & 14.7 & 18.5 \\
\hline
\end{tabular}

${ }^{1}$ Following administration of insulin

Table Ia. Serum growth hormone and blood glucose concentration following IV administration of $0.1 \mathrm{U} / \mathrm{kg}$ insulin to normal children

\begin{tabular}{|c|c|c|c|c|c|c|}
\hline \multirow{2}{*}{ Patient } & \multicolumn{6}{|c|}{ Time in minutes } \\
\hline & $02^{1}$ & 15 & 30 & 45 & 60 & 90 \\
\hline \multirow[t]{2}{*}{ BJ } & $11.3^{2}$ & - & 28.4 & - & 17.6 & 13.7 \\
\hline & $85.8^{3}$ & 63.3 & 50.0 & 68.3 & 71.7 & 83.3 \\
\hline \multirow[t]{2}{*}{$\mathrm{SC}$} & 14.2 & - & 3.4 & - & 3.4 & 2.7 \\
\hline & 82.0 & 53.0 & 37.0 & 62.1 & 71.2 & 79.5 \\
\hline \multirow[t]{2}{*}{ DB } & 9.9 & 一 & 8.1 & - & 11.0 & - \\
\hline & 69.0 & - & 39.0 & - & 47.0 & - \\
\hline \multirow[t]{2}{*}{ GG } & 5.4 & - & 5.5 & - & 14.0 & - \\
\hline & 70.8 & 一 & 33.3 & - & 70.8 & - \\
\hline \multirow[t]{2}{*}{$\mathrm{RD}$} & 9.0 & - & 3.5 & - & 4.7 & 4.5 \\
\hline & 82.3 & 53.0 & 37.1 & 62.1 & 71.2 & 79.5 \\
\hline \multirow[t]{2}{*}{ DK } & 10.7 & - & 6.2 & - & 7.2 & - \\
\hline & 98.0 & - & 73.0 & - & 84.0 & 一 \\
\hline \multirow[t]{2}{*}{ WJ } & 2.6 & - & 11.0 & - & 5.0 & 7.4 \\
\hline & 80.0 & 55.4 & 44.5 & 65.6 & 74.2 & 78.0 \\
\hline \multirow[t]{2}{*}{$\mathrm{JC}$} & 2.0 & - & 1.5 & - & 13.6 & - \\
\hline & 80.0 & - & 43.0 & - & 89.0 & - \\
\hline \multirow[t]{2}{*}{$\mathrm{IG}$} & 2.9 & 一 & 4.9 & - & 12.2 & - \\
\hline & 79.0 & - & 45.0 & - & 55.0 & 一 \\
\hline \multirow[t]{2}{*}{ NA } & 14.7 & - & 18.5 & - & 10.8 & 一 \\
\hline & 85.0 & - & 35.8 & - & 75.0 & - \\
\hline
\end{tabular}

1 At time of administration of insulin

${ }^{2}$ Upper line: $\mathrm{SGH}(\mathrm{m} \mu \mathrm{g} / \mathrm{ml})$

${ }^{3}$ Lower line: blood glucose $(\mathrm{mg} / 100 \mathrm{ml}$ ) 
Table II. Clinical data of children with hypopituitarism

\begin{tabular}{|c|c|c|c|c|c|c|c|c|c|c|c|}
\hline Patient & years & Sex & $\mathrm{cm}$ & SD & $\begin{array}{l}\text { Bone } \\
\text { age } \\
\text { years }\end{array}$ & $\begin{array}{l}\text { Growth } \\
\text { rate } \\
\mathrm{cm} / \text { year }\end{array}$ & $\begin{array}{l}\text { TSH } \\
\text { function }\end{array}$ & $\begin{array}{l}\text { ACTH } \\
\text { function }\end{array}$ & $\begin{array}{l}\text { Diabetes } \\
\text { insipidus }\end{array}$ & Diagnosis & $\begin{array}{l}\text { Maximum } \\
\text { level of } \\
\text { SGH }\end{array}$ \\
\hline $\mathrm{SC}$ & $2-4 / 12$ & $\mathrm{~m}$ & 97.0 & +2.0 & $3-6 / 12$ & 3.9 & abnormal & abnormal & present & optic glioma & 1.0 \\
\hline EW & $4-4 / 12$ & $\mathrm{f}$ & 107.4 & -1.2 & $4-0 / 12$ & 4.1 & normal & abnormal & present & craniopharyngioma & 1.0 \\
\hline WS & $6-3 / 12$ & $\mathrm{~m}$ & 116.0 & +0.9 & n.a. & 2.0 & normal & normal & present & retinoblastoma & 1.0 \\
\hline CL & $7-1 / 12$ & $\mathrm{f}$ & 106.3 & -4.5 & $5-6 / 12$ & n.a. & abnormal & abnormal & present & craniopharyngioma & 5.0 \\
\hline $\mathrm{KG}$ & $8-0 / 12$ & $\mathrm{f}$ & 115.2 & -3.0 & $6-10 / 12$ & n.a. & abnormal & abnormal & present & aberrant pinealoma & 1.0 \\
\hline $\mathrm{JR}$ & $8-5 / 12$ & $f$ & 115.5 & -2.8 & $4-2 / 12$ & 1.0 & abnormal & abnormal & present & craniopharyngioma & 2.2 \\
\hline JB & $8-8 / 12$ & $\mathrm{~m}$ & 127.1 & -3.3 & $5-0 / 12$ & 2.3 & abnormal & abnormal & present & craniopharyngioma & 1.0 \\
\hline WD & $11-9 / 12$ & $\mathrm{~m}$ & 138.5 & -2.0 & $9-6 / 12$ & 3.7 & abnormal & abnormal & present & craniopharyngioma & 1.0 \\
\hline WR & $11-9 / 12$ & $\mathrm{~m}$ & 126.8 & -3.9 & $7-0 / 12$ & 4.2 & normal & normal & present & neurofibromatosis & 1.9 \\
\hline $\mathrm{EF}$ & $12-0 / 12$ & $f$ & 146.7 & -1.0 & $10-6 / 12$ & 7.2 & normal & normal & present & craniopharyngioma & 1.0 \\
\hline JG & $12-10 / 12$ & $\mathrm{f}$ & 130.8 & -4.4 & $10-6 / 12$ & 2.3 & abnormal & abnormal & present & craniopharyngioma & 1.0 \\
\hline BS & $13-2 / 12$ & $f$ & 137.0 & -2.8 & $11-0 / 12$ & n.a. & normal & normal & present & neurofibromatosis & 1.0 \\
\hline $\mathrm{JT}$ & $14-0 / 12$ & $\mathrm{~m}$ & 141.0 & -3.0 & $10-6 / 12$ & n.a. & abnormal & abnormal & present & optic glioma & 1.0 \\
\hline MS & $14-1 / 12$ & $f$ & 136.0 & -4.5 & $9-0 / 12$ & 2.0 & abnormal & abnormal & present & craniopharyngioma & 1.0 \\
\hline DS & $15-6 / 12$ & $\mathrm{~m}$ & 145.6 & -4.0 & $13-6 / 12$ & 2.7 & abnormal & abnormal & present & craniopharyngioma & 1.0 \\
\hline LK & $7-8 / 12$ & $f$ & 117.0 & -1.9 & $6-6 / 12$ & 3.0 & abnormal & abnormal & present & histiocytosis $^{2}$ & 1.0 \\
\hline $\mathrm{CH}$ & $11-5 / 12$ & $f$ & 124.5 & -3.6 & $10-0 / 12$ & 4.1 & abnormal & normal & present & unknown ${ }^{2}$ & 1.0 \\
\hline JA & $12-8 / 12$ & $f$ & 152.2 & -1.0 & $11-6 / 12$ & 2.0 & abnormal & abnormal & present & unknown $^{2}$ & 1.0 \\
\hline
\end{tabular}

${ }^{1}$ Following administration of insulin; 1.0 indicates $\leq 1.0 \mathrm{~m} \mu \mathrm{g} / \mathrm{ml}$

${ }^{2}$ No demonstrable tumor by roentgenogram of skull and/or pneumoencephalogram

SD: Number of standard deviations from mean value of height for age

n. a.: not available 
Table II a. Serum growth hormone and blood glucose concentration following IV administration of $0.1 \mathrm{U} / \mathrm{kg}$ insulin to children with hypopituitarism

\begin{tabular}{|c|c|c|c|c|c|c|c|c|}
\hline \multirow[t]{2}{*}{ Patient } & \multicolumn{8}{|c|}{ Time in minutes } \\
\hline & $01^{1}$ & $02^{2}$ & 15 & 30 & 45 & 60 & 90 & 120 \\
\hline \multirow[t]{2}{*}{$\mathrm{SC}^{5}$} & & $1.0^{6}$ & 1.0 & 1.0 & 1.0 & 1.0 & 一 & 1.0 \\
\hline & & $87.0^{7}$ & 48.0 & 47.0 & 49.0 & 57.0 & - & 62.0 \\
\hline \multirow[t]{2}{*}{$\mathrm{EW}^{4}$} & & 1.0 & 1.0 & 1.0 & 1.0 & 1.0 & 1.0 & 1.0 \\
\hline & & 99.5 & 69.0 & 44.0 & 87.0 & 76.0 & 89.0 & 78.0 \\
\hline \multirow[t]{2}{*}{5} & & 1.0 & - & 1.0 & - & 1.0 & 1.0 & - \\
\hline & & 73.0 & 40.0 & 37.0 & - & 46.0 & 50.0 & - \\
\hline \multirow[t]{2}{*}{$\mathrm{WS}^{5}$} & & $<1.0$ & $<1.0$ & $<1.0$ & - & $<1.0$ & $<1.0$ & - \\
\hline & & 84.0 & 65.0 & 56.0 & - & 58.0 & $61.0^{3}$ & - \\
\hline \multirow[t]{2}{*}{$\mathrm{CL}^{4}$} & & 3.4 & 4.8 & 5.1 & $\cdots$ & 5.5 & $<1.0$ & $<1.0$ \\
\hline & & 48.6 & 33.8 & 31.3 & - & 31.1 & 31.8 & 42.4 \\
\hline \multirow[t]{2}{*}{$\mathrm{KC}^{5}$} & & 1.0 & - & 1.0 & - & 1.0 & 1.0 & 1.0 \\
\hline & & n.a. & $-\ldots \ldots$ & n.a. & $-\ldots \ldots$ & n.a. & n.a. & n.a. \\
\hline \multirow[t]{2}{*}{$\mathrm{JR}^{4}$} & & 1.7 & 2.0 & 2.0 & 2.2 & 1.0 & 1.4 & 1.0 \\
\hline & & 79.6 & 46.7 & 58.3 & 75.0 & 75.8 & 78.3 & 82.5 \\
\hline \multirow[t]{2}{*}{5} & & 1.7 & 2.0 & 2.0 & 2.2 & 1.4 & 1.7 & - \\
\hline & & 50.0 & 10.0 & 11.0 & 27.0 & 45.0 & 54.0 & - \\
\hline \multirow[t]{2}{*}{$\mathrm{JB}^{5}$} & & 1.0 & - & 1.0 & - & 1.0 & - & - - \\
\hline & & 74.0 & - & 54.0 & - & 57.0 & - & - \\
\hline \multirow[t]{2}{*}{$\mathrm{WD}^{5}$} & & 1.0 & 1.0 & 1.0 & 1.0 & 1.0 & 1.0 & 1.0 \\
\hline & & 82.2 & 40.9 & 38.6 & 54.7 & 57.6 & 74.2 & 73.5 \\
\hline \multirow[t]{2}{*}{$\mathrm{WR}^{5}$} & & 1.0 & - & 1.9 & - & 1.0 & 1.0 & 1.0 \\
\hline & & 85.0 & 35.6 & 30.3 & 63.6 & 76.5 & 80.3 & 87.1 \\
\hline \multirow[t]{2}{*}{$\mathrm{EF}^{4}$} & & 1.0 & 1.0 & 1.0 & - & 1.0 & 1.0 & - \\
\hline & & 97.0 & 59.0 & 39.0 & - & 62.0 & 81.0 & - \\
\hline \multirow[t]{2}{*}{$\mathrm{JG}^{5}$} & & 1.0 & 1.0 & - & 1.0 & 1.0 & 1.0 & - \\
\hline & & 74.0 & 51.0 & - & 36.0 & 38.0 & 28.0 & - \\
\hline \multirow[t]{2}{*}{$\mathrm{BS}^{4}$} & & 1.0 & - & 1.0 & - & 1.0 & 1.0 & - \\
\hline & & 103.0 & - & 64.0 & - & 45.0 & - & - \\
\hline \multirow[t]{2}{*}{$\mathrm{JT}^{4}$} & & 1.0 & 1.0 & 1.0 & 1.0 & 1.0 & 1.0 & 1.0 \\
\hline & & 81.1 & 43.5 & 28.2 & 46.8 & 53.9 & 75.8 & 78.2 \\
\hline \multirow[t]{2}{*}{$\mathrm{MS}^{5}$} & 1.0 & 1.0 & 1.0 & 1.0 & 1.0 & 1.0 & 1.0 & 1.0 \\
\hline & 74.0 & 74.0 & 51.0 & 30.0 & 34.0 & 38.0 & 46.0 & 56.0 \\
\hline \multirow[t]{2}{*}{$\mathrm{DS}^{4}$} & & 1.0 & 1.0 & 1.0 & 1.0 & 1.0 & 1.0 & - \\
\hline & & 71.7 & 62.5 & 50.0 & 69.1 & 70.8 & 80.8 & - \\
\hline \multirow[t]{2}{*}{ LK } & 1.7 & 1.0 & 1.0 & 1.6 & 1.0 & 2.9 & 1.4 & $<1.0$ \\
\hline & 69.0 & 72.0 & 50.0 & 23.0 & 38.0 & 43.0 & 55.0 & 66.0 \\
\hline \multirow[t]{2}{*}{$\dddot{\mathrm{CH}}$} & 1.2 & 1.0 & - & 1.0 & - & 1.0 & 1.0 & 1.0 \\
\hline & 82.0 & 80.0 & - & 50.0 & - & 67.0 & 72.0 & 79.0 \\
\hline \multirow[t]{2}{*}{ JA } & 1.0 & 1.0 & 1.0 & 1.0 & 1.0 & 1.0 & 1.0 & 1.0 \\
\hline & 83.0 & 83.0 & 36.0 & 20.0 & 40.0 & 45.0 & 58.0 & 78.0 \\
\hline
\end{tabular}

1 15-30 minutes before administration of insulin 1.0 indicates $<1.0 \mathrm{~m} \mu \mathrm{g} / \mathrm{ml}$

${ }^{2}$ At time of administration of insulin

${ }^{3}$ Sample taken at 75 minutes

4 Pediat. Res., Vol. 2, No. 1 (1968)
${ }^{4}$ Preoperative period

${ }^{5}$ Postoperative period

6 Upper line: SGH $(\mathrm{m} \mu \mathrm{g} / \mathrm{ml})$

${ }^{7}$ Lower line: blood glucose $(\mathrm{mg} / 100 \mathrm{ml})$ 
not differ from that observed for the control group ( $p>0.1$ ) (fig. $1 \mathrm{~b}$, tables $\mathrm{V}$ and $\mathrm{Va}$ ).

In the 5 patients with delayed adolescence, the response $(11.8 \mathrm{~m} \mu \mathrm{g} / \mathrm{ml})$ was comparable to that observed in the control group ( $p>0.2$ ) (fig. $1 \mathrm{~b}$, tables $\mathrm{V}$ and $\mathrm{Va}$ ).

Four of the 5 infants with the maternal deprivation syndrome showed evidence of increased insulin sensitivity possibly secondary to diminished stores of liver glycogen, although the fasting blood glucose was normal. The mean SGH concentration was $16.7 \mathrm{~m} \mu \mathrm{g} / \mathrm{ml}$, a value comparable to that of the control group (fig. $1 \mathrm{~b}$, table VI). One patient (JC) had a FSGH level of 3.5 $\mathrm{m} \mu \mathrm{g} / \mathrm{ml}$ which did not rise following insulin-induced hypoglycemia. All patients had a marked acceleration in height following hospitalization. None was retested after discharge from the hospital (tables VI and VIa).

In the 9 psychosocial dwarfs, a mean concentration of GH of $10.9 \mathrm{~m} \mu \mathrm{g} / \mathrm{ml}$, a value comparable to that in the control group, was demonstrated (fig. $1 \mathrm{~b}$, tables VI and VIa). Two children (RS, RR) had an abnormal response to insulin-induced hypoglycemia. In them, the FSGH concentration was $1 \mathrm{~m} \mu \mathrm{g} / \mathrm{ml}$; this failed to rise after insulin-induced hypoglycemia. RS was retested after 2 months of hospitalization and had a FSGH level of $2.0 \mathrm{~m} \mu \mathrm{g} / \mathrm{ml}$, with a maximum rise to. $7.4 \mathrm{~m} \mu \mathrm{g} / \mathrm{ml}$; intravenous infusion of arginine was used as the stimulation test, though no response to arginine infusion was demonstrable on admission. Three of the 9 patients had evidence of increased insulin sensitivity, although the fasting blood glucose in all was within the normal range. No other evidence of pituitary dysfunction was found.

Among the group with miscellaneous disorders, there was a short, pudgy 13-year-old prepubertal male (NF) who had a maximum SGH rise of $3 \mathrm{~m} \mu \mathrm{g} / \mathrm{ml}$ in response to insulin-induced hypoglycemia. His growth rate increased following administration of $\mathrm{HGH}$ but to a lesser degree than is frequently observed in hypopituitary dwarfs. He may represent an early phase of idiopathic GH deficiency. In a prepubertal male with renal and gastrointestinal anomalies (MB) and a blunted response to hypoglycemia, idiopathic $\mathrm{GH}$ deficiency could not be excluded. In a female patient (DG) with Cushing's syndrome secondary to bilateral adrenal

Table III. Clinical data of children with constitutional shortness of stature

\begin{tabular}{|c|c|c|c|c|c|c|c|c|c|}
\hline Patien & years & Sex & $\begin{array}{l}\text { Birth } \\
\text { weight } \\
\text { gm }\end{array}$ & $\begin{array}{l}\text { Height } \\
\mathrm{cm}\end{array}$ & $\mathrm{SD}$ & $\begin{array}{l}\text { Bone age } \\
\text { years }\end{array}$ & $\begin{array}{l}\text { Growth } \\
\text { rate } \\
\mathrm{cm} / \text { year }\end{array}$ & $\begin{array}{l}\text { Level of } \\
\text { fasting } \\
\mathrm{m} \mu \mathrm{g} / \mathrm{ml}\end{array}$ & $\begin{array}{l}\text { maximum } \\
\mathrm{m} \mu \mathrm{g} / \mathrm{ml}\end{array}$ \\
\hline $\mathrm{BP}$ & $2-5 / 12$ & $\mathrm{~m}$ & 2562 & 79.0 & -4.0 & $2-6 / 12$ & n.a. & 9.8 & 10.0 \\
\hline JR & $4-3 / 12$ & $\mathrm{~m}$ & 3069 & 92.6 & -3.4 & $3-0 / 12$ & 5.9 & 2.0 & 9.5 \\
\hline $\mathrm{MH}$ & $4-6 / 12$ & $\mathrm{~m}$ & 2870 & 92.2 & -3.9 & $4-6 / 12$ & 5.9 & 1.9 & 7.6 \\
\hline EM & $5-0 / 12$ & $\mathrm{~m}$ & 3462 & 104.5 & -2.5 & $3-0 / 12$ & 5.4 & 3.2 & 11.0 \\
\hline $\mathrm{RR}$ & $5-6 / 12$ & $\mathrm{~m}$ & 2307 & 99.5 & -3.3 & $4-0 / 12$ & 8.8 & 1.7 & 3.2 \\
\hline $\mathrm{BD}$ & $6-1 / 12$ & f & 2619 & 104.3 & -2.9 & $4-0 / 12$ & 6.3 & 4.4 & 14.0 \\
\hline WG & $7-4 / 12$ & $\mathrm{~m}$ & 2477 & 109.5 & -3.5 & $5-0 / 12$ & 8.4 & 15.2 & 17.2 \\
\hline $\mathrm{AG}$ & $7-8 / 12$ & $\mathrm{~m}$ & 2449 & 103.0 & -3.4 & $7-6 / 12$ & 4.8 & 1.8 & 19.4 \\
\hline $\mathrm{RP}$ & $8-0 / 12$ & $\mathrm{~m}$ & 2307 & 108.0 & -4.5 & $5-0 / 12$ & n.a. & 10.1 & 12.4 \\
\hline JW & $8-7 / 12$ & $\mathrm{~m}$ & 2814 & 116.9 & -3.0 & $6-6 / 12$ & 3.8 & 1.0 & 22.4 \\
\hline GM & $8-10 / 12$ & $\mathrm{~m}$. & 2027 & 114.3 & -3.6 & $5-6 / 12$ & 8.8 & 3.2 & 6.4 \\
\hline $\mathrm{MH}$ & $9-1 / 12$ & $\mathrm{~m}$ & 3657 & 113.1 & -3.7 & $6-0 / 12$ & n.a. & $<1.0$ & 6.7 \\
\hline WC & $10-0 / 12$ & $f$ & 2420 & 118.0 & -3.6 & $7-0 / 12$ & 4.5 & $<1.0$ & 6.9 \\
\hline $\mathrm{MJ}$ & $10-0 / 12$ & $\mathrm{~m}$ & 2812 & $\cdots 128.0$ & -2.5 & $8-6 / 12$ & 6.5 & 2.0 & 15.4 \\
\hline $\mathrm{RF}$ & $10-2 / 12$ & $\mathrm{~m}$ & 2676 & 124.2 & -3.6 & $9-0 / 12$ & n.a. & 1.0 & 24.3 \\
\hline TD & $10-10 / 12$ & $\mathrm{~m}$ & 3264 & 124.4 & -2.0 & $6-0 / 12$ & 4.4 & 7.0 & 20.0 \\
\hline $\mathrm{JF}$ & $11-2 / 12$ & $\mathrm{~m}$ & 2927 & 126.5 & -3.4 & $8-0 / 12$ & 5.2 & 2.3 & 21.5 \\
\hline $\mathrm{RT}$ & $12-0 / 12$ & $\mathrm{~m}$ & n.a. & 126.0 & -3.7 & $12-0 / 12$ & 6.0 & 1.0 & 4.4 \\
\hline JB & $12-3 / 12$ & $\mathrm{~m}$ & 2785 & 129.5 & -3.6 & $10-0 / 12$ & 4.5 & 4.7 & 11.2 \\
\hline ST & $13-5 / 12$ & $\mathrm{~m}$ & 3150 & 132.4 & -3.5 & $11-0 / 12$ & 3.3 & 5.2 & 6.8 \\
\hline
\end{tabular}

${ }^{1}$ Following administration of insulin

$\mathrm{SD}$ : Number of standard deviations from mean value of height for age n.a.: not available 
Table III a. Scrum growth hormone and blood glucose concentration following IV adm:nistration cf $0.1 \mathrm{U}, \mathrm{kg}$ insulin to children with constitutional shortness of stature

\begin{tabular}{|c|c|c|c|c|c|c|c|c|}
\hline \multirow[t]{2}{*}{ Patient } & \multicolumn{8}{|c|}{ Time in minutes } \\
\hline & $01^{1}$ & $02^{2}$ & 15 & 30 & 45 & 60 & 90 & 120 \\
\hline \multirow[t]{2}{*}{$\mathrm{BP}$} & & $9.8^{3}$ & 3.3 & 6.7 & 一 & 7.0 & 10.0 & - \\
\hline & & $92.0^{4}$ & 73.0 & 52.0 & - & 62.0 & 65.0 & - \\
\hline \multirow[t]{2}{*}{$J R$} & & 2.0 & - & 9.5 & - & 3.7 & - & -- \\
\hline & & 75.0 & - & 50.0 & - & 73.0 & - & -- \\
\hline \multirow[t]{2}{*}{$\mathrm{MH}$} & & 1.9 & 1.8 & 7.6 & - & 4.7 & 4.0 & - \\
\hline & & 97.0 & 37.0 & 54.0 & - & 57.0 & $7 \mathrm{~J} .0$ & - \\
\hline \multirow[t]{2}{*}{ EM } & & 3.2 & 5.0 & 5.0 & - & 2.5 & 11.0 & - \\
\hline & & 94.0 & 10.0 & 45.0 & - & 63.0 & 70.0 & - \\
\hline \multirow[t]{2}{*}{$\mathrm{RR}$} & 1.5 & 1.7 & $\ldots$ & 2.2 & 1.0 & 3.2 & 2.2 & 一 \\
\hline & 68.0 & 66.0 & 28.0 & 36.0 & 52.0 & 54.0 & 53.0 & $\ldots \ldots$. \\
\hline \multirow[t]{2}{*}{$\mathrm{BD}$} & - & 4.4 & 4.0 & 14.0 & 5.5 & 5.0 & 3.4 & 4.6 \\
\hline & 77.0 & 67.0 & 37.0 & 55.0 & 68.0 & 68.0 & 74.0 & 79.0 \\
\hline \multirow[t]{2}{*}{ WC } & & 15.2 & 17.5 & 17.2 & - & 11.0 & 10.4 & - \\
\hline & & 81.0 & 26.0 & 32.0 & 36.0 & 43.0 & 56.0 &.-- \\
\hline \multirow[t]{2}{*}{$\Lambda \mathrm{G}$} & & 1.8 & 6.5 & 16.5 & - & 19.4 & 0.0 & $\cdots-$ \\
\hline & & 82.0 & 52.0 & 53.0 & - & 75.0 & 75.0 & $\ldots$ \\
\hline \multirow[t]{2}{*}{$\mathrm{RP}^{\mathrm{P}}$} & - & 10.1 & 4.0 & 12.4 & - & 4.2 & 10.2 & $\ldots$ \\
\hline & 95.0 & 105.0 & 105.0 & 71.0 & 58.0 & 53.0 & 42.0 & - \\
\hline \multirow[t]{2}{*}{ JW } & & 1.0 & - & 22.4 & - & 7.5 & $\cdots$ & - \\
\hline & & 97.0 & - & 44.0 & - & 83.0 & - & --- \\
\hline \multirow[t]{2}{*}{$\mathrm{CM}$} & & 3.6 & 1.0 & 3.0 & - & 4.2 & 1.0 & 2.7 \\
\hline & & 60.0 & 34.0 & 41.0 & - & 54.0 & 61.0 & 63.0 \\
\hline \multirow[t]{2}{*}{$\mathrm{MH}$} & & $<1.0$ & - & 2.7 & - & 6.7 & 1.7 & - \\
\hline & & 80.0 & - & 66.0 & $-\ldots$ & 76.0 & 90.0 & - \\
\hline \multirow[t]{2}{*}{ WC } & & $<1.0$ & - & 6.9 & - & 6.0 & - & 1.0 \\
\hline & & 82.0 & 28.1 & 57.0 & 68.0 & 79.7 & 82.2 & $\varepsilon 2.0$ \\
\hline \multirow[t]{2}{*}{ MJ } & 2.0 & $<1.0$ & $<1.0$ & 9.5 & - & 15.0 & 15.0 & 15.4 \\
\hline & 81.0 & 78.0 & 47.0 & 43.0 & 60.0 & 64.0 & 80.0 & 92.0 \\
\hline \multirow[t]{2}{*}{$\mathrm{RF}$} & & 1.0 & $\cdots$ & 10.5 & - & 5.4 & 24.3 & - \\
\hline & & 96.0 & 49.0 & 86.0 & 87.0 & 87.0 & 89.0 & 91.0 \\
\hline \multirow[t]{2}{*}{ 'I'D } & & 8.0 & 12.5 & 20.0 & - & 16.0 & 12.0 & - \\
\hline & & 88.0 & 46.0 & 43.0 & - & 51.0 & 80.0 & $\cdots-$ \\
\hline \multirow[t]{2}{*}{$\mathrm{JF}$} & & 2.3 & - & 2.4 & 4.6 & 14.5 & 21.5 & - \\
\hline & & 74.0 & - & 28.0 & $30.0^{\circ}$ & 33.0 & 40.0 & - \\
\hline \multirow[t]{2}{*}{$\mathrm{R}^{\prime} \mathrm{I}$} & & 1.7 & 1.8 & 1.4 & - & 4.4 & 4.1 & - \\
\hline & & 80.0 & 46.0 & 32.0 & $-\ldots$ & 66.0 & 75.0 & - \\
\hline \multirow[t]{2}{*}{ JB } & 4.7 & 5.9 & 11.2 & 8.8 & 9.6 & 5.4 & 5.8 & 2.4 \\
\hline & 79.0 & 79.0 & 27.0 & 71.0 & 79.0 & 95.0 & 79.0 & 30.0 \\
\hline \multirow[t]{2}{*}{$\mathrm{ST}$} & 4.6 & 5.2 & 2.0 & 6.8 & 5.5 & 5.8 & 4.0 & 3.4 \\
\hline & 74.0 & 66.0 & 44.0 & - & 53.0 & 60.0 & 66.0 & 66.0 \\
\hline
\end{tabular}

15-30 minutes before administration of insulin

2 At time of administration of insulin

${ }^{3}$ Upper line: $\mathrm{SGH}(\mathrm{m} / \mathrm{g} / \mathrm{ml})$

Lower line: blood glucose $(\mathrm{mg} / 100 \mathrm{mI})$ 
hyperplasia, the maximum response to insulin-induced hypoglycemia before adrenalectomy was $2.5 \mathrm{~m} \mu \mathrm{g} / \mathrm{ml}$; resumption of menses and a growth spurt of 10-12 cm was observed within a year following bilateral adrenalectomy. Two patients had evidence of chronic disease of prolonged duration, 2 had hematologic disorders, 2 had symptoms of malabsorption, and one had growth retardation associated with obesity but without evidence of Cushing's syndrome or a hypothalamic tumor (table VII). The mean maximum serum response of this group was $7.0 \mathrm{~m} \mu \mathrm{g} / \mathrm{ml}$ (fig. $1 \mathrm{~b}$, tables VII and VIIa).

When the SGH data for the entire group of children with growth retardation were analyzed by the logarithmic transformation method, there was a highly significant difference in the response of the control group compared to the group with hypopituitarism (p $<0.001)$. The response to insulin-induced hypoglycemia by children with other forms of growth retardation, excluding the nonclassified group, was not significantly different from the response observed in the control group ( $p>0.5$ ) (fig. $1 \mathrm{a}$ and $1 \mathrm{~b}$ ).

\section{Factors Affecting Serum Growth Hormone Response to Insulin-} Induced Hypoglycemia

a) Fasting serum growth hormone concentration. It became evident during these studies that excitement alone could induce a marked increase in the response. The fasting levels were elevated in some of the children during periods of excitement (tables I-VI). In 18 children the FSGH was more than $9.0 \mathrm{~m} \mu \mathrm{g} / \mathrm{ml}$. A decrease from the fasting concentration or no response

Table IV. Clinical data of children with primordial dwarfism

\begin{tabular}{|c|c|c|c|c|c|c|c|c|c|}
\hline Patien & years & Sex & $\begin{array}{l}\text { Birth } \\
\text { weight } \\
\text { gm }\end{array}$ & $\begin{array}{l}\text { Height } \\
\mathrm{cm}\end{array}$ & $\mathrm{SD}$ & $\begin{array}{l}\text { Bone age } \\
\text { years }\end{array}$ & $\begin{array}{l}\text { Growth } \\
\text { rate } \\
\mathrm{cm} / \text { year }\end{array}$ & $\begin{array}{l}\text { Level of } \\
\text { fasting } \\
\mathrm{m} \mu \mathrm{g} / \mathrm{ml}\end{array}$ & $\begin{array}{l}\mathrm{m} \\
\operatorname{maximum} \\
\mathrm{m} \mu \mathrm{g} / \mathrm{ml}\end{array}$ \\
\hline $\mathrm{JP}$ & $7 / 12$ & $f$ & 2506 & 57.0 & -4.0 & $3 / 12$ & 18.8 & 8.8 & 16.0 \\
\hline $\mathrm{KS}$ & $8 / 12$ & $f$ & 2250 & 56.8 & -4.5 & $3 / 12$ & n.a. & 2.8 & 6.2 \\
\hline $\mathrm{RP}^{2}$ & $9 / 12$ & $\mathrm{~m}$ & 2141: & 55.0 & -5.5 & $3 / 12$ & 14.1 & 7.8 & 16.5 \\
\hline $\mathrm{KT}^{2}$ & $1-4 / 12$ & $\mathrm{~m}$ & 2676 & 67.3 & -3.9 & $6 / 12$ & 13.0 & 2.6 & 10.2 \\
\hline $\mathrm{ER}^{2}$ & $2-7 / 12$ & $\mathrm{~m}$ & 1127 & 69.4 & -5.9 & $1-6 / 12$ & 4.0 & 12.0 & 17.5 \\
\hline WK & $3-1 / 12$ & $\mathrm{~m}$ & 4050 & 86.5 & -3.0 & $2-0 / 12$ & 7.5 & 1.0 & 19.5 \\
\hline AM & $3-1 / 12$ & $\mathrm{~m}$ & n.a. & 80.0 & -4.7 & $2-8 / 12$ & n.a. & 3.2 & 8.0 \\
\hline ET & $4-4 / 12$ & $f$ & 1856 & 90.0 & -4.0 & $2-6 / 12$ & 6.8 & 13.7 & 10.4 \\
\hline $\mathrm{MG}$ & $4-9 / 12$ & $f$ & 900 & 92.2 & -3.9 & $4-6 / 12$ & n.a. & 7.6 & 12.5 \\
\hline $\mathrm{JK}$ & $5-6 / 12$ & $f$ & n.a. & 100.0 & -3.0 & $3-6 / 12$ & n.a. & 1.0 & 7.0 \\
\hline GG & $5-10 / 12$ & $\mathrm{~m}$ & 2112 & 102.3 & -3.4 & $3-6 / 12$ & 7.2 & 24.0 & 15.0 \\
\hline$W^{3} W^{3}$ & $6-3 / 12$ & $f$ & 1662 & 99.0 & -3.0 & $6-6 / 12$ & 5.4 & 1.0 & 21.0 \\
\hline EF & $6-7 / 12$ & f & 3117 & 104.0 & -3.6 & $3-6 / 12$ & 3.3 & 1.0 & 9.2 \\
\hline $\mathrm{JM}^{4}$ & $8-0 / 12$ & $\mathrm{~m}$ & 2720 & 104.5 & -4.8 & $8-0 / 12$ & 3.3 & 5.2 & 4.7 \\
\hline $\mathrm{DH}$ & $8-7 / 12$ & $\mathrm{~m}$ & 3685 & 112.5 & -3.5 & $4-0 / 12$ & n.a. & 4.8 & 5.5 \\
\hline$Y P^{3}$ & $9-9 / 12$ & $\mathrm{~m}$ & 2000 & 112.2 & -5.2 & $6-0 / 12$ & 1.6 & 1.0 & 7.0 \\
\hline $\mathrm{SH}$ & $10-0 / 12$ & $f$ & 2250 & 111.5 & -5.0 & $6-6 / 12$ & 3.5 & 1.0 & 9.2 \\
\hline MT & $10-2 / 12$ & $\mathrm{f}$ & 2250 & 111.4 & -5.3 & $5-0 / 12$ & 4.0 & 5.2 & 26.5 \\
\hline $\mathrm{MH}$ & $10-3 / 12$ & $\mathrm{~m}$ & n.a. & 123.8 & -3.3 & $6-6 / 12$ & n.a. & 1.0 & 13.6 \\
\hline $\mathrm{MH}$ & $10-10 / 12$ & $\mathrm{f}$ & 2169 & 124.0 & -3.6 & $10-6 / 12$ & 6.0 & 2.4 & 10.7 \\
\hline $\mathrm{DA}$ & $12-2 / 12$ & $\mathrm{~m}$ & 3264 & 134.5 & -2.6 & 8-0/12 & 4.2 & 1.0 & 10.0 \\
\hline $\mathrm{CG}^{3}$ & $15-0 / 12$ & $\mathrm{~m}$ & 3714 & 130.0 & -5.6 & $10-9 / 12$ & n.a. & 11.1 & 16.4 \\
\hline
\end{tabular}

${ }^{1}$ Following administration of insulin

${ }^{2}$ Russell-Silver

${ }^{3}$ Bird-headed

${ }^{4}$ Progeria

SD: Number of standard deviations from mean value of height for age

n. a. : not available
Footnotes for table IV a:

1 15-30 minutes before administration of insulin

${ }^{2}$ At time of administration of insulin

${ }^{3}$ Test discontinued, symptoms of hypoglycemia

${ }^{4}$ Upper line: $\mathrm{SGH}(\mathrm{m} \mu \mathrm{g} / \mathrm{ml})$

${ }^{5}$ Lower line: blood glucose $(\mathrm{mg} / 100 \mathrm{ml})$ 
Growth and growth hormone

Table IVa. Serum growth hormone and blood glucose concentration following IV administration of $0.1 \mathrm{U} / \mathrm{kg}$ insulin to children with primordial dwarfism

\begin{tabular}{|c|c|c|c|c|c|c|c|c|}
\hline \multirow[t]{2}{*}{ Patient } & \multicolumn{8}{|c|}{ Time in minutes } \\
\hline & $01^{1}$ & $02^{2}$ & 15 & 30 & 45 & 60 & 90 & 120 \\
\hline \multirow[t]{2}{*}{ JP } & & $8.8^{4}$ & 7.7 & 16.0 & 8.3 & 10.2 & 14.5 & - \\
\hline & & $69.0^{5}$ & 22.0 & 26.0 & 34.0 & 38.0 & 69.0 & - \\
\hline \multirow[t]{2}{*}{$\mathrm{KS}$} & & 2.8 & 2.6 & $6.2^{3}$ & & & & \\
\hline & & 74.0 & 15.0 & 43.0 & & & & \\
\hline \multirow[t]{2}{*}{$\mathrm{RP}$} & & 7.8 & - & 6.0 & - & 8.7 & 16.5 & - \\
\hline & & 56.0 & - & 39.0 & - & 29.0 & 44.0 & - \\
\hline \multirow[t]{2}{*}{ KT } & & 2.4 & 2.1 & 8.4 & 5.7 & 4.6 & 2.6 & 1.8 \\
\hline & & 69.0 & 36.0 & 48.0 & 61.0 & 70.0 & 71.0 & 70.0 \\
\hline \multirow[t]{2}{*}{ ER } & & 12.0 & - & 7.7 & - & 17.0 & 14.7 & 11.0 \\
\hline & & 45.0 & 37.0 & 39.0 & 42.0 & 42.0 & 55.0 & 66.0 \\
\hline \multirow[t]{2}{*}{ WK } & & 1.0 & - & 6.4 & $-\cdots$ & 19.5 & - & - \\
\hline & & 83.0 & - & 37.0 & - & 47.0 & - & - \\
\hline \multirow[t]{2}{*}{$\mathrm{AM}$} & & 3.2 & 1.1 & 3.0 & - & 8.0 & 6.4 & 3.5 \\
\hline & & 60.0 & 34.0 & 41.0 & - & 54.0 & 61.0 & 63.0 \\
\hline \multirow[t]{2}{*}{ ET } & & 13.7 & - & 10.4 & - & 8.3 & 8.0 & 9.0 \\
\hline & & 80.0 & - & 41.0 & - & 56.0 & 67.0 & 67.0 \\
\hline \multirow[t]{2}{*}{$\mathrm{MC}$} & & 7.6 & 3.3 & 12.5 & - & 9.1 & 5.0 & 2.2 \\
\hline & & 74.0 & 38.0 & 37.0 & - & 59.0 & 68.0 & 62.0 \\
\hline \multirow[t]{2}{*}{$\mathrm{JK}$} & & 1.0 & - & 7.0 & - & 5.0 & 4.8 & - \\
\hline & & 89.0 & 39.0 & 46.0 & 54.0 & 60.0 & 70.0 & - \\
\hline \multirow[t]{2}{*}{$\mathrm{GG}$} & & 24.0 & - & 15.0 & $-\cdots$ & 7.4 & $\cdots$ & 1.0 \\
\hline & & 79.0 & 51.0 & 46.0 & 64.0 & 61.0 & 72.0 & 73.0 \\
\hline \multirow[t]{2}{*}{ Ww } & & $<1.0$ & - & 21.0 & - & 10.0 & - & - \\
\hline & & 60.8 & - & 63.3 & - & 75.0 & - & - \\
\hline \multirow[t]{2}{*}{$\mathrm{EF}$} & & 1.0 & - & 3.2 & - & 9.2 & 5.7 & 3.7 \\
\hline & & 79.0 & - & 39.0 & - & 68.0 & 77.0 & 87.0 \\
\hline \multirow{2}{*}{$\mathrm{JM}$} & 4.7 & 5.2 & 2.2 & 3.2 & 1.8 & 2.0 & 4.4 & 4.7 \\
\hline & 76.0 & 73.0 & 32.0 & 53.0 & 63.0 & 74.0 & 80.0 & 76.0 \\
\hline \multirow[t]{2}{*}{$\mathrm{DH}$} & & 4.8 & - & 5.5 & - & 2.5 & - & - \\
\hline & & 88.0 & - & 46.0 & - & 88.0 & - & - \\
\hline \multirow[t]{2}{*}{$\mathrm{YP}$} & 1.0 & 2.8 & - & 5.4 & - & 4.8 & 6.4 & 7.0 \\
\hline & 78.0 & 73.0 & 52.0 & 54.0 & 74.0 & 81.0 & 81.0 & 100.0 \\
\hline \multirow[t]{2}{*}{$\mathrm{SH}$} & 1.0 & 1.0 & 1.0 & 2.0 & 6.0 & 9.2 & 5.0 & 2.1 \\
\hline & 81.0 & 83.0 & 44.0 & 34.0 & 51.0 & 56.0 & 72.0 & 76.0 \\
\hline \multirow[t]{2}{*}{$\mathrm{MT}$} & & 5.2 & - & 26.5 & - & 15.5 & 1.0 & 1.0 \\
\hline & & 64.0 & 17.0 & 48.0 & 66.0 & 93.0 & 91.0 & 92.0 \\
\hline \multirow[t]{2}{*}{$\mathrm{MH}$} & 2.8 & 2.4 & 3.0 & 4.0 & - & 8.6 & 10.7 & 7.5 \\
\hline & 80.0 & 76.0 & 48.0 & 40.0 & - & 57.0 & 67.0 & 76.0 \\
\hline \multirow[t]{2}{*}{ МH } & & 1.9 & 1.8 & 7.6 & - & 4.7 & 4.0 & - \\
\hline & & 97.0 & 37.0 & 54.0 & - & 57.0 & 70.0 & - \\
\hline $\mathrm{DA}$ & $<1.0$ & $<1.0$ & $<1.0$ & 3.7 & 9.3 & 8.2 & 10.0 & - \\
\hline & 78.0 & 80.0 & 28.0 & 39.0 & 65.0 & 75.0 & 83.0 & - \\
\hline$\dddot{\mathrm{CC}}$ & & 11.1 & 3.0 & 7.0 & 8.0 & 10.0 & 14.5 & 10.7 \\
\hline & & 81.0 & 36.0 & 32.0 & 49.0 & - & 57.0 & 60.0 \\
\hline
\end{tabular}




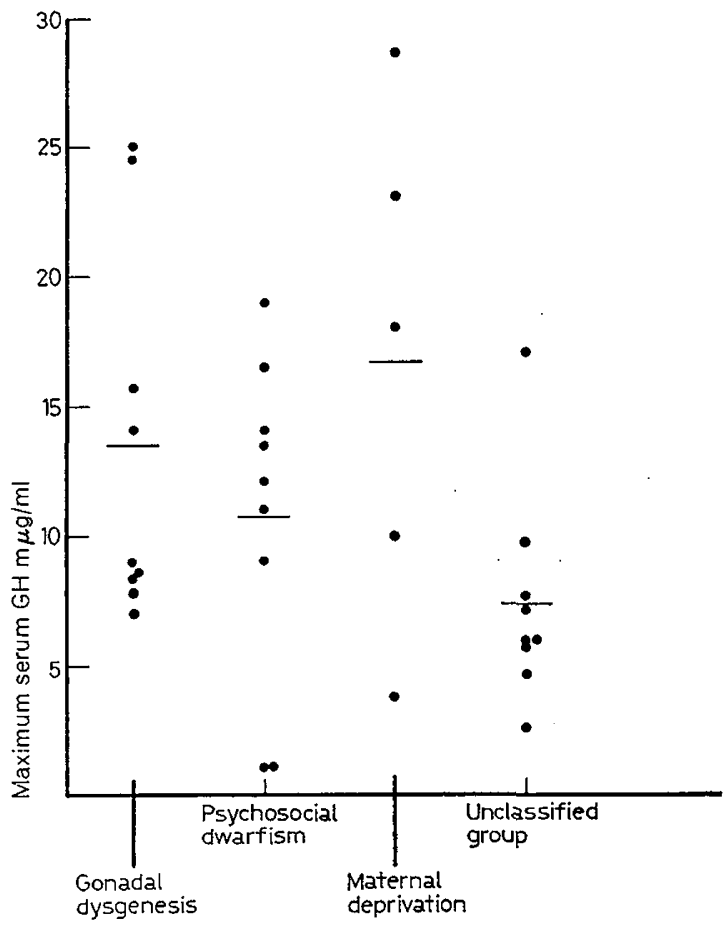

was observed in 8 patients ( 4 of whom were in the control group) following insulin-induced hypoglycemia. Five patients responded by a rise of $5 \mathrm{~m} \mu \mathrm{g} / \mathrm{ml}$ or less, and 5 showed almost a two-fold increase above the fasting concentration following insulin induced hypoglycemia. There was no greater frequency of this type of response in the younger children-age distribution of the 18 children was 6 months to 15 years. This 'paradoxical response' was not characteristic of any particular form of growth retardation nor was it observed more often in hyperactive or young children.

b) Age and sex. The FSGH level was significantly higher in children $<4$ years of age (mean $6.6 \mathrm{~m} \mu \mathrm{g} / \mathrm{ml}$ ), especially in those $<2$ years (mean $7.9 \mathrm{~m} \mu \mathrm{g} / \mathrm{ml}$ ) in comparison with older subjects. Analysis by the chi square method revealed a $\mathrm{p}$ value of $<0.001$. There

Fig. 1b. The maximum SGH concentration following insulin-induced hypogylcemia is indicated as $\mathrm{m} \mu \mathrm{g} / \mathrm{ml}$. The horizontal line designates the mean level for the group. No difference is observed in mean concentration of children with gonadal dysgenesis, psychosocial dwarfism, or maternal deprivation. Note that only 2 of 9 patients with psychosocial dwarfism had responses similar to that of patients with GH deficiency.

Table $V$. Clinical data on children with delayed adolescence and gonadal dysgenesis

\begin{tabular}{|c|c|c|c|c|c|c|c|c|c|}
\hline Patient & $\begin{array}{l}\text { Age } \\
\text { years }\end{array}$ & Sex & $\begin{array}{l}\text { Birth. } \\
\text { weight } \\
\text { gm }\end{array}$ & $\begin{array}{l}\text { Height } \\
\mathrm{cm}\end{array}$ & SD & $\begin{array}{l}\text { Bone age } \\
\text { years }\end{array}$ & $\begin{array}{l}\text { Growth } \\
\text { rate } \\
\mathrm{cm} / \text { year }\end{array}$ & $\begin{array}{l}\text { Level of } \\
\text { fasting } \\
\mathrm{m} \mu \mathrm{g} / \mathrm{ml}\end{array}$ & $\begin{array}{l}\operatorname{maximum}^{1} \\
\mathrm{~m} \mu \mathrm{g} / \mathrm{ml}\end{array}$ \\
\hline \multicolumn{10}{|l|}{$\begin{array}{l}\text { Dclayed } \\
\text { adolescencc }\end{array}$} \\
\hline $\mathrm{DB}$ & $13-1 / 12$ & $f$ & 3406 & 143.3 & -2.6 & $11-0 / 12$ & 3.7 & 1.4 & 5.7 \\
\hline GL & $13-3 / 12$ & $\mathrm{~m}$ & 5376 & 130.0 & -3.7 & $8-0 / 12$ & 3.1 & 1.7 & 10.0 \\
\hline $\mathrm{KD}$ & $13-4 / 12$ & $f$ & 2477. & 142.0 & -3.0 & $9-6 / 12$ & n.a. & 1.0 & 5.6 \\
\hline $\mathrm{RR}$ & $13-8 / 12$ & $\mathrm{~m}$ & n.a. & 139.6 & -2.8 & $11-0 / 12$ & n.a. & 2.5 & 16.0 \\
\hline HW & $14-9 / 12$ & $\mathrm{~m}$ & 3060 & 129.0 & -5.3 & $10-0 / 12$ & 4.5 & 11.4 & 21.5 \\
\hline \multicolumn{10}{|l|}{$\begin{array}{l}\text { Gonadal } \\
\text { dysgenesis }\end{array}$} \\
\hline MA & $6-1 / 12$ & $f$ & 3320 & 103.3 & -3.2 & $6-0 / 12$ & 4.6 & 2.3 & 15.8 \\
\hline $\mathrm{PE}$ & $8-9 / 12$ & f & 2307 & 118.0 & -3.0 & $8-0 / 12$ & n.a. & 1.0 & 24.5 \\
\hline $\mathrm{EO}$ & $8-9 / 12$ & $f$ & 3264 & 116.3 & -3.4 & $8-6 / 12$ & 4.9 & 12.0 & 25.0 \\
\hline CM & $11-6 / 12$ & $f$ & 2757 & 122.8 & -4.6 & $11-0 / 12$ & 4.0 & 3.5 & 7.0 \\
\hline RE & $12-0 / 12$ & $f$ & 2477 & 122.0 & -4.6 & $8-0 / 12$ & 3.6 & 1.0 & 9.0 \\
\hline VV & $12-2 / 12$ & f & 2449 & 123.0 & -4.7 & $8-6 / 12$ & 4.1 & 3.2 & 8.4 \\
\hline PA & $12-6 / 12$ & f & 2927 & 122.5 & -5.3 & $12-0 / 12$ & 3.2 & 10.6 & 7.9 \\
\hline OD & $12-6 / 12$ & $f$ & n.a. & 135.0 & -3.4 & $12-0 / 12$ & n.a. & 1.0 & 8.6 \\
\hline GL & $14-3 / 12$ & f & 2392 & 130.5 & -5.7 & $11-0 / 12$ & 3.5 & 1.5 & 14.0 \\
\hline
\end{tabular}

${ }^{1}$ Following administration of insulin

SD: Number of standard deviations from mean value of height for age

n.a.: not available 
was no apparent correlation of the FSGH level with age in children 4 to 16 years of age (mean $4.0 \mathrm{~m} \mu \mathrm{g} / \mathrm{ml}$ ) (fig.2). No sex differences were observed.

c) Blood glucose. A decrease in blood glucose concentration to levels of 40 to $60 \%$ of the fasting value was achieved in all but 2 patients. There was no significant difference in the maximal GH response achieved in the group with a 40 to $50 \%$ decrease in blood glucose, compared with those with a 50 to $60 \%$ decrease in blood glucose, or with the group with $60 \%$ or more decrease in blood glucose following administration of intravenous insulin.

Response to Therapy with Human Pituitary Growth Hormone Eight children who had a SGH response of $3 \mathrm{~m} \mu \mathrm{g} / \mathrm{ml}$ or more were treated for a period of 6 months to a year with $\mathrm{HGH}$ in doses of 2-4 mg 3 times a week. Growth data on all children for at least 6 months before and

Table Va. Serum growth hormone and blood glucose concentration following IV administration of $0.1 \mathrm{U} / \mathrm{kg}$ insulin to children with delayed adolescence and gonadal dysgenesis

\begin{tabular}{|c|c|c|c|c|c|c|c|}
\hline \multirow[t]{2}{*}{ Patient } & \multicolumn{7}{|c|}{ Time in minutes } \\
\hline & $02^{1}$ & 15 & 30 & 45 & 60 & 90 & 120 \\
\hline \multicolumn{8}{|c|}{ Gonadal dysgenesis } \\
\hline \multirow[t]{2}{*}{ MA } & $2.3^{3}$ & - & 2.4 & 一 & 15.8 & - & 一 \\
\hline & $73.6^{4}$ & 一 & 40.2 & - & 50.0 & - & 一 \\
\hline \multirow[t]{2}{*}{ PE } & 1.0 & - & 24.5 & - & 17.0 & $\cdots$ & - \\
\hline & 79.0 & $-\ldots$ & 50.0 & - & 78.0 & - & - \\
\hline \multirow[t]{2}{*}{$\mathrm{EO}$} & 12.0 & - & 25.0 & 一 & 9.5 & - & - \\
\hline & 77.0 & - & 36.0 & - & 66.0 & - & - \\
\hline \multirow[t]{2}{*}{ GM } & 3.5 & 3.5 & 7.0 & - & $4.0^{2}$ & 一. & - \\
\hline & 82.0 & 50.0 & 37.0 & - & 57.0 & 一 & - \\
\hline \multirow[t]{2}{*}{$\mathrm{RE}$} & 1.0 & - & 9.0 & - & 3.5 & $\ldots$ & $<1.0$ \\
\hline & 72.0 & 58.0 & 46.0 & 66.0 & 71.0 & 69.0 & 72.0 \\
\hline \multirow[t]{2}{*}{ VV } & 3.2 & 1.2 & 2.6 & 8.4 & 6.8 & 4.3 & - \\
\hline & 86.0 & 34.0 & $46.0^{\circ}$ & 92.0 & 92.0 & 94.0 & - \\
\hline \multirow[t]{2}{*}{ PA } & 10.6 & - & 7.9 & - & 6.3 & - & - \\
\hline & 88.8 & - & 29.1 & - & 70.0 & - & - \\
\hline \multirow[t]{2}{*}{ OD } & 1.0 & - & 7.6 & - & - & 8.6 & - \\
\hline & 83.0 & 63.0 & 28.0 & - & 85.0 & 68.0 & - \\
\hline \multirow[t]{2}{*}{ GL } & 1.5 & - & 14.0 & 一... & 6.6 & $\ldots$ & - \\
\hline & 69.2 & - & 41.7 & 一 & 63.3 & - & - \\
\hline \multicolumn{8}{|c|}{ Delayed adolescence } \\
\hline \multirow[t]{2}{*}{ DB } & 1.4 & - & 3.5 & 一 & 5.4 & 5.7 & - \\
\hline & 92.5 & 53.3 & 40.0 & 83.3 & 80.1 & 88.3 & - \\
\hline \multirow[t]{2}{*}{ GL } & 1.7 & - & $10.0^{5}$ & & & & \\
\hline & 92.0 & 42.0 & 44.0 & & & & \\
\hline \multirow[t]{2}{*}{$\mathrm{KD}$} & 1.0 & 1.0 & 5.6 & - & 3.1 & 1.2 & - \\
\hline & 73.0 & 20.0 & 43.0 & - & 63.0 & 82.0 & - \\
\hline \multirow[t]{2}{*}{$\mathrm{RR}$} & 2.5 & - & 16.0 & - & 9.0 & 6.0 & - \\
\hline & 76.0 & - & 27.0 & - & 68.0 & 78.0 & - \\
\hline \multirow[t]{2}{*}{ HW } & 11.4 & — & 21.5 & - & 10.0 & 4.8 & 5.8 \\
\hline & 86.0 & - & 44.0 & - & 60.0 & 82.0 & 82.0 \\
\hline
\end{tabular}

1 At time of administration of insulin

${ }^{2}$ Sample taken at 75 minutes

${ }^{3}$ Upper line: SGH $(\mathrm{m} \mu \mathrm{g} / \mathrm{ml})$
${ }^{4}$ Lower line: blood glucose $(\mathrm{mg} / 100 \mathrm{ml})$

${ }^{5}$ Test discontinued, symptoms of hypoglycemia 
after therapy were examined. Increments in height for the periods before, during and after treatment with $\mathrm{HGH}$ are given in table VIII. In 6 patients there was no significant change in rate of growth during the period of $\mathrm{HGH}$ administration. Three (DB, MB, KT) had a low titer $(<0.1 \mu \mathrm{g} / \mathrm{ml} \mathrm{HGH}$ binding capacity) of antibodies to HGH detectable at the termination of therapy, and 3 (JR, CM, MT) had no detectable antibodies during or within 6 months after therapy. One patient (ER) had an acceleration in his growth rate during the period of treatment, but subsequent reinstitution of therapy failed to elicit a similar growth response. Serum antibodies to $\mathrm{HGH}$ of $<0.1 \mu \mathrm{g} / \mathrm{ml}$ binding capacity were detected during the first course of therapy, with no increase during the second period.

In another patient (NF) there was an apparent increase in the growth rate from a height increment of $4.9 \mathrm{~cm} /$ year before therapy to $7.0 \mathrm{~cm} /$ year during the treatment period. This decreased to $3.5 \mathrm{~cm} /$ year when therapy was discontinued. Serum antibodies to $\mathrm{HGH}$ were detected at a level of $<1 \mu \mathrm{g} / \mathrm{ml}$ binding affinity at the termination of therapy. During the second year following discontinuation of $\mathrm{HGH}$, his growth rate increased to $6.0 \mathrm{~cm} /$ year associated with early signs of pubertal development. In contrast, 20 patients with plasma GH levels of $<2 \mathrm{~m} \mu \mathrm{g} / \mathrm{ml}$ before and after insulin-induced hypoglycemia had a growth rate of 9 to $16 \mathrm{~cm} /$ year during the initial phase of treatment with a dose of $2 \mathrm{mg} 3$ times a week.

\section{Discussion}

These studies have failed to provide evidence of $\mathrm{GH}$ deficiency or abnormality in the responsiveness of the hypothalamic growth hormone-releasing mechanism [32] to insulin-induced hypoglycemia in most children with a wide variety of growth disorders, other than hypopituitary dwarfism. All had been growing at a steady rate, although the degree of retardation in

Table VI. Clinical data on children with maternal deprivation syndrome and psychosocial dwarfism

\begin{tabular}{|c|c|c|c|c|c|c|c|c|c|c|}
\hline \multirow[t]{2}{*}{ Patient } & \multirow{2}{*}{$\begin{array}{l}\text { Age } \\
\text { years }\end{array}$} & \multirow[t]{2}{*}{ Sex } & \multirow{2}{*}{$\begin{array}{l}\text { Birth } \\
\text { weight } \\
\text { gm }\end{array}$} & \multirow{2}{*}{$\begin{array}{l}\text { Height } \\
\mathrm{cm}\end{array}$} & \multirow[t]{2}{*}{$\mathrm{SD}$} & \multirow{2}{*}{$\begin{array}{l}\text { Bone age } \\
\text { years }\end{array}$} & \multicolumn{2}{|c|}{ Growth rate } & \multicolumn{2}{|c|}{ Level of SGH } \\
\hline & & & & & & & $\begin{array}{l}\text { pre }^{1} \\
\mathrm{~cm} / \text { year }\end{array}$ & $\begin{array}{l}\text { post }^{2} \\
\mathrm{~cm} / \text { year }\end{array}$ & $\begin{array}{l}\text { fasting } \\
\mathrm{m} \mu \mathrm{g} / \mathrm{ml}\end{array}$ & $\begin{array}{l}\operatorname{maximum}^{3} \\
\mathrm{~m} \mu \mathrm{g} / \mathrm{ml}\end{array}$ \\
\hline \multicolumn{11}{|c|}{$\begin{array}{l}\text { Maternal } \\
\text { deprivation }\end{array}$} \\
\hline DM & $6 / 12$ & $\mathrm{~m}$ & 2899 & 59.5 & -3.0 & $3 / 12$ & n.a. & 20.0 & 10.0 & 9.9 \\
\hline LH & $1-1 / 12$ & $\mathrm{~m}$ & 2757 & 68.5 & -4.5 & $9 / 12$ & n.a. & 6.0 & 17.0 & 28.8 \\
\hline $\mathrm{JC}$ & $1-1 / 12$ & $\mathrm{~m}$ & 1212 & 65.0 & -3.4 & $6 / 12$ & n.a. & 13.0 & 3.9 & 3.8 \\
\hline $\mathrm{MC}$ & $1-1 / 12$ & $f$ & 2250 & 70.0 & -3.3 & n.a. & n.a. & 20.0 & 13.6 & 23.0 \\
\hline DW & $1-7 / 12$ & $\mathrm{~m}$ & 3126 & 67.0 & -6.0 & $3 / 12$ & n.a. & 13.0 & 6.0 & 18.0 \\
\hline \multicolumn{11}{|c|}{$\begin{array}{l}\text { Psychosocial } \\
\text { dwarfism }\end{array}$} \\
\hline DB & $3-1 / 12$ & $\mathrm{~m}$ & 3377 & 78.2 & -6.3 & $1-6 / 12$ & 1.2 & 9.3 & 4.2 & 14.2 \\
\hline DP & $4-2 / 12$ & $\mathrm{~m}$ & 3434 & 93.0 & -3.2 & $3-0 / 12$ & n.a. & 5.5 & 1.4 & 19.0 \\
\hline $\mathrm{RS}$ & $4-6 / 12$ & $\mathrm{~m}$ & 2757 & 82.5 & -6.0 & $2-6 / 12$ & 2.0 & 25.0 & 1.0 & 1.0 \\
\hline $\mathrm{MB}$ & $5-5 / 12$ & $\mathrm{~m}$ & 3207 & 85.3 & -6.7 & $3-0 / 12$ & 3.9 & 10.3 & 3.2 & 9.2 \\
\hline $\mathrm{KD}$ & $5-6 / 12$ & $\mathrm{~m}$ & 3519 & 94.0 & -4.4 & $2-6 / 12$ & n.a. & 9.6 & 4.0 & 11.0 \\
\hline $\mathrm{JZ}$ & $5-9 / 12$ & $\mathrm{~m}$ & 3742 & 87.6 & -5.9 & $2-0 / 12$ & 2.0 & 11.5 & 4.3 & 16.6 \\
\hline $\mathrm{TM}$ & $6-2 / 12$ & $\mathrm{~m}$ & 1350 & 97.2 & -4.8 & $2-8 / 12$ & n.a. & 12.3 & 2.0 & 12.2 \\
\hline $\mathrm{RR}$ & $7-8 / 12$ & $\mathrm{~m}$ & 3178 & 100.0 & -5.0 & $4-6 / 12^{4}$ & 2.2 & n.a. & 1.0 & 1.0 \\
\hline $\mathrm{DE}$ & $10-0 / 12$ & $f$ & 960 & 114.0 & -4.8 & $8-0 / 12$ & 3.8 & 14.0 & 1.0 & 13.6 \\
\hline
\end{tabular}

\footnotetext{
1 Prehospitalization period

${ }^{2}$ Posthospitalization period

${ }^{3}$ Following administration of insulin

${ }^{4}$ At $6-11 / 12$ years

SD: Number of standard deviations from mean value of height for age n. a.: not available
} 
Table VIa. Serum growth hormone and blood glucose concentration following IV administration of $0.1 \mathrm{U} / \mathrm{kg}$ insulin to children with maternal deprivation syndrome and psychosocial dwarfism

\begin{tabular}{|c|c|c|c|c|c|c|c|c|}
\hline \multirow[t]{2}{*}{ Patient } & \multicolumn{8}{|c|}{ Time in minutes } \\
\hline & $01^{1}$ & $02^{2}$ & 15 & 30 & 45 & 60 & 90 & 120 \\
\hline \multicolumn{9}{|c|}{$\begin{array}{l}\text { Maternal } \\
\text { deprivation }\end{array}$} \\
\hline \multirow[t]{2}{*}{$\mathrm{DM}$} & & $10.0^{4}$ & 9.2 & $9.9^{3}$ & & & & \\
\hline & & $57.0^{5}$ & 13.0 & 13.0 & & & & \\
\hline \multirow[t]{2}{*}{ LH } & & 17.0 & - & 15.5 & - & 28.8 & 19.2 & 14.6 \\
\hline & & 87.0 & 80.0 & 52.0 & 37.0 & 56.0 & 64.0 & 92.0 \\
\hline \multirow[t]{2}{*}{$\mathrm{JC}$} & & 3.9 & 3.7 & 3.0 & - & 3.8 & 3.6 & - \\
\hline & & 63.0 & 23.0 & 14.0 & - & 42.0 & 39.0 & - \\
\hline \multirow[t]{2}{*}{$\mathrm{MC}$} & & 10.0 & - & 23.0 & - & - & 19.4 & - \\
\hline & & 81.0 & 26.0 & 32.0 & 36.0 & 43.0 & 56.0 & - \\
\hline \multirow[t]{2}{*}{ DW } & & 6.0 & - & 15.0 & - & 18.0 & 5.2 & 9.2 \\
\hline & & 73.0 & 25.0 & 33.0 & 40.0 & 40.0 & 43.0 & 44.0 \\
\hline \multicolumn{9}{|c|}{$\begin{array}{l}\text { Psychosocial } \\
\text { dwarfism }\end{array}$} \\
\hline \multirow[t]{2}{*}{$\mathrm{DB}$} & & 4.2 & 一 & 14.2 & - & 3.8 & 3.7 & 一 \\
\hline & & 122.0 & 81.0 & 40.0 & 66.0 & 65.0 & 70.0 & - \\
\hline \multirow[t]{2}{*}{$\mathrm{DP}$} & 1.4 & 1.0 & 19.0 & 7.8 & - & 2.4 & 1.0 & 10.0 \\
\hline & 83.0 & 83.0 & 24.0 & 46.0 & - & 46.0 & 59.0 & 61.0 \\
\hline \multirow[t]{2}{*}{$\mathrm{RS}$} & 1.0 & 1.0 & - & $1.0^{3}$ & & & & \\
\hline & 74.0 & 77.0 & - & 30.0 & & & & \\
\hline \multirow[t]{4}{*}{ MB } & & 3.2 & - & 5.0 & - & 8.0 & 5.0 & - \\
\hline & & 77.4 & 29.3 & 47.4 & 49.1 & 56.9 & 77.6 & - \\
\hline & & 4.7 & - & 4.0 & - & 7.4 & 5.5 & - \\
\hline & & 90.0 & 39.0 & 47.0 & 62.0 & 64.0 & 66.0 & - \\
\hline \multirow[t]{2}{*}{$\mathrm{KD}$} & & 4.0 & 一 & 11.0 & - & 3.0 & 2.4 & 1.6 \\
\hline & & 78.0 & - & 50.0 & - & 64.0 & 74.0 & 78.0 \\
\hline \multirow[t]{2}{*}{$\mathrm{JZ}$} & & 4.3 & - & 16.6 & - & 10.4 & 2.0 & - \\
\hline & & 71.5 & 28.0 & 33.0 & 54.0 & 40.0 & 53.0 & 48.0 \\
\hline \multirow[t]{2}{*}{$\mathrm{TM}$} & 1.9 & 2.0 & 1.4 & 9.8 & 5.8 & 12.2 & 3.2 & 2.6 \\
\hline & 77.0 & 75.0 & 49.0 & 37.0 & 57.0 & 58.0 & 66.0 & 73.0 \\
\hline \multirow[t]{2}{*}{$\mathrm{RR}$} & 1.0 & 1.0 & - & 1.0 & 1.0 & 1.0 & 1.0 & 1.0 \\
\hline & 75.0 & 70.0 & - & 36.0 & 56.0 & 63.0 & 71.0 & 72.0 \\
\hline \multirow[t]{2}{*}{$\mathrm{DE}$} & & 4.4 & - & 2.5 & 5.6 & 13.6 & & \\
\hline & & 92.0 & 52.0 & 30.0 & $58.0^{3}$ & 151.0 & & \\
\hline
\end{tabular}

1 15-30 minutes before administration of insulin

2 At time of administration of insulin

3 Test discontinued, symptoms of hypoglycemia

${ }^{4}$ Upper line: SGH $(\mathrm{m} \mu \mathrm{g} / \mathrm{ml})$

${ }^{5}$ Lower line: blood glucose $(\mathrm{mg} / 100 \mathrm{ml})$ 
height varied from -2 to $-7 \mathrm{SD}$ from the mean value for height for their age. On the basis of our data, a FSGH level of $7 \mathrm{~m} \mu \mathrm{g} / \mathrm{ml}$ or an increase to $7 \mathrm{~m} \mu \mathrm{g} / \mathrm{ml}$ or more following insulin-induced hypoglycemia has a high degree of probability for association with normal pituitary acidophile function. A borderline rise of 3-5 $\mathrm{m} \mu \mathrm{g} / \mathrm{ml}$ may be the first evidence of GH deficiency. These criteria make it possible to distinguish, in most instances, children with GH deficiency from those patients with other growth disorders. However, it is evident from our experience with provocative tests for the assessment of $\mathrm{GH}$ secretion that a rigid definition of the normal range of SGH response to hypoglycemia cannot be established, because in a small proportion of instances, there is overlap between the normal subject and some patients with GH deficiency.

Difficulties in the interpretation of the $\mathrm{GH}$ response have been encountered. Certain disorders, including hypothyroidism, glucocorticoid excess, psychosocial dwarfism and obesity, may modify the GH response to insulin-induced hypoglycemia. Furthermore, discrepancies have been observed in the response of a given child to different provocative tests. Two children in this series (RR, JM) had a moderate increase in SGH above $20 \mathrm{~m} \mu \mathrm{g} / \mathrm{ml}$ following infusion of arginine, but a blunted response following hypoglycemia. Blunted responses to arginine have also been observed in children with a normal rise associated with hypoglycemia [37]. Tests repeated at intervals of 3 months in a limited number of children have demonstrated a remarkable individual consistency in the magnitude of response to a single stimulus. Children who show a limited rise in $\mathrm{SGH}$ levels following insulin-induced hypoglycemia should be examined for response following administration of arginine [22] or vasopressin [5] to corroborate this finding. In some children who have an apparently normal serum 'immunoreactive' $\mathrm{GH}$ response to a provocative test despite physical stigmata of hypopitui-

Table VII. Clinical data on children with miscellaneous disorders associated with growth retardation

\begin{tabular}{|c|c|c|c|c|c|c|c|c|c|c|}
\hline \multirow[t]{2}{*}{ Patient } & \multirow{2}{*}{$\begin{array}{l}\text { Age } \cdot \\
\text { years }\end{array}$} & \multirow[t]{2}{*}{ Sex } & \multirow{2}{*}{$\begin{array}{l}\text { Birth } \\
\text { weight } \\
\text { gm }\end{array}$} & \multirow{2}{*}{$\begin{array}{l}\text { Height } \\
\mathrm{cm}\end{array}$} & \multirow{2}{*}{$\mathrm{SD}$} & \multirow{2}{*}{$\begin{array}{l}\text { Bone } \\
\text { age } \\
\text { years }\end{array}$} & \multirow{2}{*}{$\begin{array}{l}\text { Growth } \\
\text { rate } \\
\mathrm{cm} / \text { year }\end{array}$} & \multirow{2}{*}{ Diagnosis } & \multicolumn{2}{|c|}{ Level of SGH } \\
\hline & & & & & & & & & $\mathrm{m} \mu \mathrm{g} / \mathrm{ml}$ & $\mathrm{m} \mu \mathrm{g} / \mathrm{ml}$ \\
\hline $\mathrm{JD}$ & $1-2 / 12$ & $\mathrm{f}$ & 2676 & 66.0 & -3.7 & $1-0 / 12$ & n.a. & Ehlers-Danlos & 5.2 & 10.8 \\
\hline TF & $1-10 / 12$ & $f$ & 2477 & 70.0 & -4.7 & $1-0 / 12$ & 15.6 & celiac syndrome & 3.8 & 4.2 \\
\hline GL & $4-0 / 12$ & $f$ & 4500 & 87.0 & -5.0 & $3-6 / 12$ & 4.8 & obesity & 2.3 & 7.6 \\
\hline $\mathrm{ET}$ & $8-11 / 12$ & $f$ & 2226 & 111.1 & -4.2 & $5-0 / 12$ & 4.9 & $\begin{array}{l}\text { idiopathic } \\
\text { thrombocytopenic } \\
\text { purpura in } \\
\text { remission }\end{array}$ & 2.6 & 5.8 \\
\hline $\mathrm{KG}$ & $10-0 / 12$ & $\mathrm{~m}$ & 3264 & 127.4 & -2.6 & $6-0 / 12$ & 5.8 & $\begin{array}{l}\text { malabsorption } \\
\text { syndrome }\end{array}$ & 2.6 & 6.0 \\
\hline MM & $10-3 / 12$ & f & n.a. & 109.0 & -5.5 & $5-0 / 12$ & n.a. & Gaucher's disease & $<1.0$ & 6.0 \\
\hline NF & $11-8 / 12$ & $\mathrm{~m}$ & 900 & 126.0 & -3.0 & $6-0 / 12$ & 3.9 & $\begin{array}{l}\text { ? partial GH } \\
\text { deficiency }\end{array}$ & 1.5 & 3.0 \\
\hline DG & $14-4 / 12$ & f & 3150 & 139.7 & -4.1 & $10-6 / 12$ & none & $\begin{array}{l}\text { Cushing's } \\
\text { syndrome }\end{array}$ & 1.0 & 2.6 \\
\hline EJ & $14-10 / 12$ & $\mathrm{~m}$ & 2619 & 131.5 & -5.3 & $10-0 / 12$ & n.a. & $\begin{array}{l}\text { cirrhosis } \\
\text { malabsorption } \\
\text { syndrome }\end{array}$ & 2.6 & 17.0 \\
\hline SB & $16-0 / 12$ & $f$ & 3041 & 142.0 & -4.5 & $12-0 / 12$ & none & $\begin{array}{l}\text { lymphoma in } \\
\text { remission }\end{array}$ & 1.7 & 9.7 \\
\hline $\mathrm{MB}$ & $16-2 / 12$ & $\mathrm{~m}$ & 3434 & 127.1 & -5.5 & $13-0 / 12$ & 3.5 & $\begin{array}{l}\text { multiple congeni- } \\
\text { tal anomalies }\end{array}$ & 2.0 & 4.6 \\
\hline
\end{tabular}

${ }^{1}$ Following administration of insulin

SD: Number of standard deviations from mean value of height for age n.a.: not available 
tarism, short term therapy with HGH may be necessary to establish the diagnosis of $\mathrm{GH}$ insufficiency.

Thyroxine secretion may also affect the growth hormone response to stimuli. Eight of 15 children with hypothyroidism who were studied had a diminished concentration of SGH in blood [12].

FSGH concentration can also influence the effect of provocative stimuli on GH secretion. In the presence of a moderate elevation $(9 \mathrm{~m} \mu \mathrm{g} / \mathrm{ml})$ of the fasting concentration, normal children as well as those with growth retardation tend to have either a less pronounced rise or a decrease following insulin-induced hypoglycemia. These observations suggest that temporary depletion of the pituitary content of GH following an initial surge of secretion may modify the response to a second stimulus, i.e., hypoglycemia. An alternate explanation may be that the elevated concentration in itself can, in some children, suppress further secretion by the pituitary through an effect on the growth hormone-releasing center in the hypothalamus. This type of response should not be misinterpreted as indicative of hypothalamic dysfunction in children with growth retardation, since a similar response has been observed in normal children. These findings are not in agreement with those of GREEN et al. [9] who ascribed this type of response to insulin-induced hypoglycemia as typical of children with cystic fibrosis. On the basis of our results, it is possible that the abnormal response observed in their study was related not to the basic disease, but rather to the elevated

Table VII a. Serum growth hormone and blood glucose concentration following IV administration of $0.1 \mathrm{U} / \mathrm{kg}$ insulin to children with miscellaneous disorders associated with growth retardation

\begin{tabular}{|c|c|c|c|c|c|c|c|}
\hline \multirow[t]{2}{*}{ Patient } & \multicolumn{7}{|c|}{ Time in minutes } \\
\hline & $02^{x}$ & 15 & 30 & 45 & 60 & 90 & 120 \\
\hline \multirow[t]{2}{*}{ JD } & $5.2^{3}$ & 一 & 10.8 & - & 5.6 & 10.8 & - \\
\hline & $79.0^{4}$ & - & 52.0 & - & 51.0 & 57.0 & - \\
\hline \multirow[t]{2}{*}{$\mathrm{TF}$} & 3.8 & - & 3.6 & 4.2 & 3.5 & 2.2 & - \\
\hline & 80.0 & - & 31.0 & 46.0 & 40.0 & 42.0 & - \\
\hline \multirow[t]{2}{*}{ CL } & 2.3 & - & 7.6 & - & 5.5 & - & - \\
\hline & 55.0 & 一 & 14.0 & - & 27.0 & - & - \\
\hline \multirow[t]{2}{*}{ ET } & 2.6 & 1.5 & 5.0 & - & 4.3 & 5.8 & - \\
\hline & 76.0 & 29.0 & 52.0 & $-\ldots$ & 51.0 & 56.0 & $-\ldots$ \\
\hline \multirow[t]{2}{*}{$\mathrm{KG}$} & 2.5 & 一 & 4.5 & - & 5.9 & 6.0 & - \\
\hline & 111.0 & 78.0 & 48.0 & 91.0 & 94.0 & 122.0 & - \\
\hline \multirow[t]{2}{*}{$\mathrm{MM}$} & 1.0 & - & 2.5 & - & 6.0 & 4.5 & - \\
\hline & 83.0 & 57.0 & 37.0 & 50.0 & 63.0 & 74.0 & 83.0 \\
\hline \multirow[t]{2}{*}{ NF } & 1.5 & - & 1.3 & - & 3.0 & 2.0 & - \\
\hline & 80.0 & 48.0 & 47.0 & 62.0 & 71.0 & 71.0 & - \\
\hline \multirow[t]{2}{*}{$\mathrm{DG}^{2}$} & 1.2 & - & 1.4 & 1.5 & 2.6 & 1.5 & 1.2 \\
\hline & 72.0 & 65.0 & 32.0 & 42.0 & 75.0 & 110.0 & 106.0 \\
\hline \multirow[t]{2}{*}{$\mathrm{EJ}$} & 2.6 & - & 10.5 & - & 17.0 & 2.0 & 2.0 \\
\hline & 96.0 & - & 57.0 & $-\ldots$ & 89.0 & 95.0 & 93.0 \\
\hline \multirow[t]{2}{*}{ SB } & 1.7 & - & 1.5 & - & 9.7 & - & 3.7 \\
\hline & 79.0 & 45.0 & 36.0 & 51.0 & 56.0 & 71.0 & 82.0 \\
\hline \multirow[t]{2}{*}{ MB } & 2.0 & 1.9 & 4.4 & - & 4.6 & 1.4 & 一 \\
\hline & 64.0 & 42.0 & 28.0 & - & 49.0 & 54.0 & - \\
\hline
\end{tabular}

1 At time of administration of insulin

$20.15 \mathrm{U}$ insulin $/ \mathrm{kg}$ administered

${ }^{3}$ Upper line: SGH $(\mathrm{m} \mu \mathrm{g} / \mathrm{ml})$

${ }^{4}$ Lower line: blood glucose $(\mathrm{mg} / 100 \mathrm{ml})$ 
FSGH concentration. Furthermore, the elevated fasting levels observed in the present study indicate that stress may be as effective a stimulus to SGH release as insulin-induced hypoglycemia. Similar observations in patients have been reported by Grick et al. [7] and in the monkey by MEYER and KNOBIL [23].

Another source of error in the interpretation of SGH response may result from premature termination of the sampling period. Although the peak response was ob-

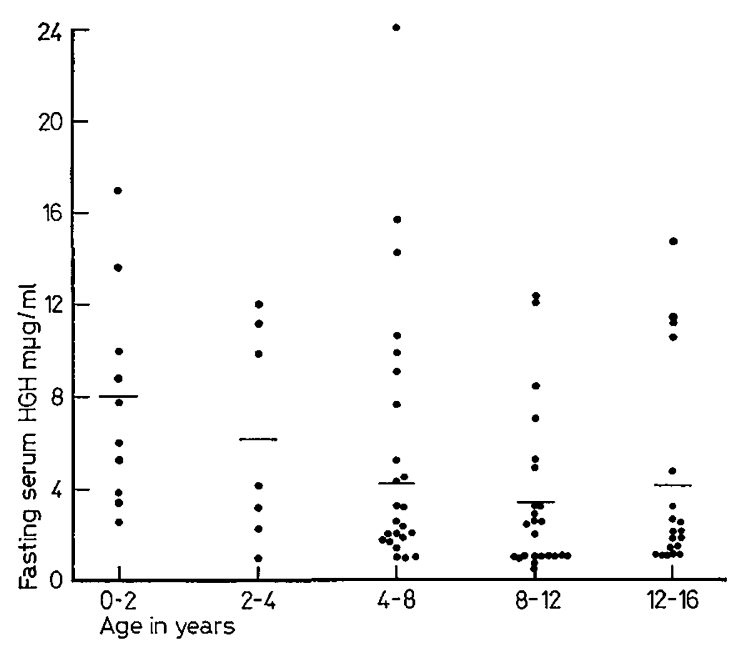

Fig. 2. The SGH concentration $(\mathrm{m} \mu \mathrm{g} / \mathrm{ml})$ in the fasting state is indicated for normal children and children with growth retardation according to age. The mean is represented by the horizontal line. Note the higher mean concentration in the children less than 4 years of age. served in most children by 60 minutes, 13 did not achieve maximum response until 90 to 120 minutes following administration of insulin. Frequent sampling at 15-minute intervals does not enhance the possibility of detecting the peak rise.

The influence of higher cerebral centers on growth is suggested by findings in children with psychosocial dwarfism. Despite a severe degree of growth retardation in this syndrome, abnormalities in $\mathrm{SGH}$ secretion were observed in only 2 of the 9 in our series, and 6 of the 10 described by Poweln et al. [24]. These children showed a marked acceleration in growth, increase in weight and personality changes following improvement in the social environment. The precise role of $\mathrm{GH}$ in the mediation of these changes was not apparent since not all had a diminution of circulating SGH.

It is noteworthy that a response to insulin-induced hypoglycemia of a lesser magnitude has been demonstrated in children in comparison with that seen in adults $[3,15,19,28]$. These observations suggest that the secretory pattern of GH may be partly dependent on the concentration of circulating gonadal hormones. Rises in GH levels in serum during the menstrual cycle coincide with the peak secretion of estrogens [2]. In the male, estrogen increases the FSGH concentration following minimal physical activity [2] and increases the SGH response to arginine infusion [22]. Furthermore, an increase in the magnitude of the response to hypoglycemia and to arginine has been observed by us following estrogen therapy in children with gonadal dysgenesis or constitutional tallness of stature. Further evidence of the possible relation of secretion of gonadal hormones to SGH is the decrease in the magnitude of response in elderly patients [18].

Table VIII. Growth rate of children with short stature following administration of HGH

\begin{tabular}{|c|c|c|c|c|c|c|c|}
\hline \multirow[t]{2}{*}{ Patient } & \multirow{2}{*}{$\begin{array}{l}\text { Age } \\
\text { years }\end{array}$} & \multicolumn{3}{|c|}{$\begin{array}{l}\text { Height increment } \\
\mathrm{cm} / \text { year }\end{array}$} & \multirow{2}{*}{$\begin{array}{l}\text { Dosage } \\
\text { of HGH } \\
\text { mg/t.i.w. }\end{array}$} & \multirow{2}{*}{$\begin{array}{l}\text { Duration of } \\
\text { administration } \\
\text { months }\end{array}$} & \multirow[t]{2}{*}{ Diagnosis } \\
\hline & & pre & during & $\overline{\text { post }}$ & & & \\
\hline $\mathrm{KT}$ & $1-4 / 12$ & 10.0 & 8.0 & 11.3 & 4 & 6 & primordial dwarfism \\
\hline \multirow[t]{2}{*}{ ER } & $2-0 / 12$ & 4.0 & 9.8 & 2.8 & 2 & 10 & primordial dwarfism \\
\hline & & 2.8 & 4.2 & n.a. & 2 & 3 & \\
\hline JR & $3-8 / 12$ & 7.4 & 6.0 & 3.7 & 4 & 6 & primordial dwarfism \\
\hline $\mathrm{DB}$ & $3-9 / 12$ & 1.2 & 10.4 & 9.3 & 2 & 8 & psychosocial dwarfism \\
\hline MB & $5-6 / 12$ & 3.9 & 8.4 & 10.3 & 2 & 8 & psychosocial dwarfism \\
\hline GM & $8-10 / 12$ & 8.8 & 9.4 & n.a. & 4 & 8 & $\begin{array}{l}\text { constitutional } \\
\text { shortness of stature }\end{array}$ \\
\hline $\mathrm{MT}$ & $10-0 / 12$ & 4.0 & 4.3 & 3.0 & 2 & 12 & primordial dwarfism \\
\hline $\mathrm{NF}$ & $13-0 / 12$ & 3.9 & 7.0 & 6.9 & 2 & 6 & possible GH deficiency \\
\hline
\end{tabular}

1 t.i.w.: 2 times per week

n. a.: not available 
Sensitivity of the hypothalamus to stimuli affecting the release of $\mathrm{GH}$ may be altered at puberty. The conversion to the adult-type response may depend not only on an increase in circulating levels of testosterone or estrogen, but also on an alteration of the responsiveness of the hypothalamus to these stimuli. In the rat, testosterone implants in the median eminence have been more effective in suppressing release of the luteinizing hormone in the prepubertal than in pubertal animals [34] and this observation suggests that modification of feedback control for the secretion of pituitary hormones may occur at puberty.

Age differences in the disposal rate for pituitary growth hormone with a more rapid turnover in children could lead to a lower concentration of SGH. This possibility must remain speculative, since no data are available on either the secretory or disposal rate of $\mathrm{GH}$ in prepubertal children.

In the present study there was no evidence that the immunoreactive GH was not also biologically active. The failure to demonstrate an acceleration in growth rate on administration of $\mathrm{HGH}$ in the children with measurable immunoreactive $\mathrm{GH}$ is in accord with this hypothesis. However, a familial form of growth retardation has been described which may be associated with the secretion of a structurally abnormal GH; affected children respond to exogenously administered pituitary GH despite elevated SGH levels [20]. None of our patients had this form of growth retardation.

The SGH response to insulin-induced hypoglycemia has been valuable in distinguishing children with $\mathrm{GH}$ deficiency, especially when it occurs as an isolated deficiency, from those with other forms of growth retardation, but it has not provided insight as to the mechanism of growth failure in other forms of growth retardation. It is recognized that use of provocative agents has limitations in the assessment of $\mathrm{GH}$ deficiency. However, by the criteria stated, it was possible to identify 16 patients with no other evidence of pituitary deficiency but in whom the diagnosis of isolated growth hormone deficiency could be made. All had a marked acceleration in growth following administration of $\mathrm{HGH}$. In contrast, 7 patients with a normal SGH response to hypoglycemia did not show a sustained growth acceleration following treatment with HGH.

It should be emphasized that one-third of the patients with hypopituitarism who were treated developed antibodies to HGH of low binding capacity which did not have any apparent effect on their growth rate during $\mathrm{HGH}$ administration [36]. These results are in sharp contrast to previously published studies [25, 33]. The low titer of HGH antibodies found in some children with growth retardation following the administration of HGH was not related to their unresponsiveness to $\mathrm{HGH}$ therapy.
A prolonged period of observation (minimum of 6 months) during and following administration of $\mathrm{HGH}$ is valuable in establishing the effectiveness of hormonal treatment in any patient. This should minimize misinterpretation of seasonal changes in growth rate or of transient acceleration of growth which may occur in patients without GH deficiency who are treated with HGH.

\section{Summary}

Changes in concentration of growth hormone in serum (SGH) following insulin-induced hypoglycemia has been evaluated in 134 children with growth retardation. On the basis of this test, a deficiency in growth hormone was demonstrable in 53 patients. Thirtyseven of these children had, in addition, evidence of deficiency of other pituitary hormones and 16 children appeared to have an isolated growth hormone deficiency. Children with primordial dwarfism, constitutional shortness of stature, gonadal dysgenesis and delayed adolescence responded to hypoglycemia with changes in levels of growth hormone in serum comparable to that observed in a control group. Difficulties in the interpretation of test responses have been discussed. Following intramuscular administration of pituitary growth hormone, an increased growth rate was observed in children with a demonstrable growth hormone deficiency, but no sustained change in growth rate was discernible in children who had a normal growth hormone response to hypoglycemia.

\section{References and Notes}

1. Berson, S.A.; Yalow, R.S.; Bauman, A.; RothSCHILD, M.A. and NEWERLY, K.: Insulin ${ }^{131}$ metabolism in human subjects: Demonstration of insulin-binding globulin in circulation of insulin treated subjects. J. clin. Invest. 35: 170 (1956).

1a. Ferguson, K.A. and Wallace, A.L.G.: The characterization of pituitary hormones by starch gel electrophoresis. Recent Progr. Hormone Res. 19: 1 (1963).

2. Frantz, A.G. and Rabkin, M.T.: Effect of estrogen and sex difference on secretion of human growth hormone. J.clin. Endocr. 25: 1470 (1965).

3. Frasier, S.D.: The serum growth hormone response to hypoglycemia in dwarfism. Western Society for Pediatric Research, Portland, Oregon (Abstract) (November 1965).

4. Frohman, L.A.; Horton, E.S. and Lebovitz, H. E. : Growth hormone releasing action of a pseu- 
domonas endotoxin (piromen). Metabolism 16: 57 (1967).

5. Gagliardino, J.J.; Bailey, J.D. and Martin, J.M.: Effect of vasopressin on serum-levels of human growth hormone. Lancet $i: 1357$ (1967).

6. Glick, S. M.; Roth, J.; Yalow, R. S. and Berson, S.A.: Immunoassay of human growth hormone in plasma. Nature (Lond.) 199: 784 (1963).

7. Glick, S. M.; Roth, J.; Yalow, R.S. and Berson, S.A.: The regulation of growth hormone secretion. Recent Progr. Hormone Res. 21: 241 (1965).

8. Goodman, H. G.; Grumbach, M.M. and Kaplan, S.L.: Growth hormone and growth. II. A comparison of isolated growth hormone deficiency and multiple pituitary hormone deficiencies in $35 \mathrm{pa}-$ tients with idiopathic hypopituitary dwarfism. New Engl.J.Med. (in press 1967).

9. Green, O.C.; Fefferman, R. and Nair, S.: Plasma growth hormone levels in children with cystic fibrosis and short stature. Unresponsiveness to hypoglycemia. J. clin. Endocr. 27: 1059 (1967).

10. Greenwood, F. C.; Hunter, W.M. and Glover, J.S.: The preparation of ${ }^{131}$ I-labeled human growth hormone of high specific radioactivity. Biochem.J. 89: 114 (1963).

11. Greulich, W.W. and Pyle, S.I.: Radiographic atlas of skeletal development of the hand and wrist, 2nd ed.(California University Press, Stanford 1959).

12. Grumbach, M. M. and Kaplan, S. L.: Unpublished observations.

13. Hunter, W.M.; Fonseka, C.C. and Passmore, $\mathrm{R}$.: The role of growth hormone in the mobilization of fuel for muscular exercise. Quart. J.exp. Physiol. 50: 406 (1965).

14. Hunter, W.M. and Greenwood, F.C.: Studies on the secretion of pituitary growth hormone. Brit. med.J. $i: 804$ (1964).

15. Kaplan, S. L. ; Abrams, C. A. L.; Bell, J.J. ; Conte F.A. and Grumbach, M.M.: Serum growth hormone: Response to insulin-induced hypoglycemia in disorders of growth. J.Pediat. (Abstract) 67:956 (1965).

16. Kaplan, S.L. and Grumbach, M.M.: Immunoassay for human chorionic 'growth hormone prolactin' in serum and urine. Science 147: 751 (1965).

17. Knopf, R.T.; Gonn, J.N.; Floyd, J. C.; GuntSCHE, E. M. and RuLl, J. A.: Plasma growth hormone response to intravenous administration of amino acids. J. clin. Endocr. 25: 1140 (1965).

18. Laron, Z. and Amikam, B.: Growth hormone (GH) secretion in old age. Endocrine Society Meetings (Abstract) (June 1967).

19. LARon, Z. and MANnhermer, S.: Measurement of human growth hormone: Description of the method. Israel J.med. Sci. 2: 115 (1966).
20. Laron, Z.; Pertzelan, A. and Mannheimer, S.: Genetic pituitary dwarfism with high serum concentration of growth hormone: A new inborn error of metabolism? Israel J.med.Sci. 2: 152 (1966).

21. Liddle, G.W.; Estep, H.L.; Kendall, J.W.; Williams, W.G. and Townes, A.W.: Clinical application of a new test of pituitary reserve. J. clin. Endocr. 19: 875 (1959).

22. Merimee, T.J.; Riggs, L.; Rimoin, D. L.; Rabinowitz, D.; Burgess, J.A. and MaKusick, V.A.: Plasma growth hormone after arginine infusion. New Engl.J. Med. 276: 434 (1967).

23. MEYer, V. and KNoBIL, E.: Growth hormone secretion in the unanesthetized rhesus monkey in response to noxious stimuli. Endocrinology 80: 163 (1967).

24. Powell, G. F.; Brasel, J. A. and Buizzard, R. M.: Emotional deprivation and growth retardation simulating hypopituitarism. I. Clinical evaluation of the syndrome. II. Endocrinologic evaluation of the syndrome. New Engl.J.Med. 276: 1271, 1279 (1967).

25. Prader, A.; Wagner, H.; Szeky, J.; Illlig, R.; Touber, J.L. and MAINGAy, D.: Acquired resistance to human growth hormone caused by specific antibodies. Lancet $i i$ : 378 (1964).

26. REED, R. B. and StUART, H. C.: Patterns of growth in height and weight from birth to eighteen years of age. Pediatrics 24: 904 (1959).

27. Richmond, L.; Ghappell, J. and Gleveland, W.W.: Response of children to methopyrapone. J.Pediat. 64: 381 (1964).

28. Root, A.W.; Rosenfield, R.L.; Bongiovanni, A.M. and Eberlein, W.R.: The plasma growth hormone response to insulin-induced hypoglycemia in children with retardation of growth. Pediatrics 39: 844 (1967).

29. Roth, J.; Glick, S. M.; Yalow, R.S. and Berson, S.A.: Secretion of human growth hormone: Physiologic and experimental modification. Metabolism 12: 577 (1963).

30. Roth, J.; Glick, S. M.; Yalow, R. S. and Berson, S. A.: Hypoglycemia. A potent stimulus to secretion of growth hormone. Science 140: 987 (1963).

31. SAifer, A. and Gerstenfeld, S.: The photometric micro determination of blood glucose with glucose oxidase. J. Lab. clin. Med. 51: 448 (1958).

32. Schally, A.V.; Muller, E.E.; Arimura, A.; Bowers, C.; SaIto, T.; Redding, T.W.; Sawano, S. and Pizzolato, P.: Releasing factors in human hypothalamic and neurohypophysial extracts. J. clin. Endocr. 27: 755 (1967).

33. SeIP, M. and Trygstad, O.: Experiences with human growth hormone in pituitary dwarfism. Acta Paediat. (Uppsala) 55: 287 (1966). 
34. Smrtr, E.R. and Davidson, J.M.: Differential responses to hypothalamic testosterone in relation to male puberty. Amer. J. Physiol. 212: 1385 (1967).

35. WiLkins, L.: The diagnosis and treatment of endocrine disorders in childhood and adolescence, 2 nd ed. (Thomas, Springfield, Ill. 1957).

36. Wolter, R.; Kaplan, S.L. and Grumbach, M. M.: Antibodies to $\mathrm{HGH}$ following administration of $\mathrm{HGH}$ to 30 children (in preparation).

37. Youlton, R.; Kaplan, S.L. and Grumbach, M.M.: Comparison of the serum growth hormone response to insulin-induced hypoglycemia and to arginine infusion in children with growth retardation (in preparation).

38. Presented in part at the Thirty-fifth Annual Meeting of the Society for Pediatric Research, Philadelphia, Pa., May 4-6, 1965.

39. We wish to thank Dr. Alfred Wilhelmi for his gift of human growth hormone used as a standard in the radioimmunoassay; The National Pituitary Agency for their generous supply of human growth hormone used in these studies; and to Miss AngeLES JARDIOLIN for technical assistance.

40. Dr. S.L. Kaplan is recipient of a Career Development Award from the National Institute of Child Health and Development. Drs. C.A.L.Abrams, J.J. Bell and F.A. Conte are Trainees in Pediatric Endocrinology under program sponsored by the National Institute of Arthritis and Metabolic Diseases. This investigation was supported by grants from the National Institute of Child Health and Human Development and by the National Institute of Arthritis and Metabolic Diseases.

41. Grumbach, M. M., M.D., Professor and Chairman, Department of Pediatrics, University of California School of Medicine, San Francisco, Cal. 94122 (USA). 